\title{
L'anthropo-sociologie poétique de Pierre Sansot: les sports et le sensible
}

Yves Le Pogam

\section{(2) OpenEdition}

1 Journals

\section{Édition électronique}

URL : http://journals.openedition.org/corpsetculture/264

DOI : 10.4000/corpsetculture.264

ISSN : $1777-5337$

Éditeur

Association Corps et Culture

\section{Édition imprimée}

Date de publication : 1 juin 1995

ISSN : 1268-5631

Référence électronique

Yves Le Pogam, «L'anthropo-sociologie poétique de Pierre Sansot : les sports et le sensible », Corps et culture [En ligne], Numéro 1 | 1995, mis en ligne le 11 mai 2007, consulté le 07 septembre 2020. URL: http://journals.openedition.org/corpsetculture/264; DOI : https://doi.org/10.4000/corpsetculture.264

Ce document a été généré automatiquement le 7 septembre 2020

(c) tous droits réservés 


\section{L'anthropo-sociologie poétique de Pierre Sansot : les sports et le sensible}

Yves Le Pogam

"La situation qui est faite aujourd'hui au langage comporte cette double possibilité, cette double sollicitation, cette double urgence. D'un côté, purifier les discours de ses excroissances, liquider les idoles, aller de l'ébriété à la sobriété, faire une bonne fois le bilan de notre pauvreté ; de l'autre côté, user du mouvement le plus "nihiliste", le plus destructeur, le plus iconoclaste, pour laisser parler ce qui une fois, ce qui chaque fois a été dit quand le sens parut neuf, quand le sens était plein ; l'herméneutique me paraît mue par cette double motivation : volonté de soupçon, volonté

d'écoute ; voeu de rigueur, voeu d'obéissance ; nous sommes aujourd'hui ces hommes qui n'ont pas fini de faire mourir les idoles et qui commencent à peine à entendre les symboles".

(Ricoeur P., 1965 : 36)

1 Il est des tâches dont on ne calcule ni l'ampleur ni les difficultés, celles entre autres de prétendre en quelques pages restituer l'essence de ce que Georges Balandier a appelé, "une vraie oeuvre, singulière et novatrice" (1993), constituée depuis une vingtaine d'années par les publications de Pierre Sansot composées d'une dizaine d'ouvrages et de très nombreux articles parus dans les revues scientifiques prestigieuses ou dans des ouvrages collectifs. Une telle entreprise solitaire comporte d'inévitables limites qui doivent être soulignées d'emblée. 
2 Certaines sont liées à la prétention de croire qu'il est possible d'extraire la réalité d'une pensée à partir de la lecture de milliers de pages, mais c'est là une réserve qui vaudrait pour quelque auteur d'importance. D'autres tiennent par contre à la spécificité de l'oeuvre elle-même, car se dégage de sa lecture une qualité émotionnelle, sentiment qui prête plus à une préservation jalouse qu'à une exposition réglée ôtant obligatoirement beaucoup de sensibilité à l'écriture originelle. Le risque est donc double ici : celui de réduire une pensée unique échafaudée patiemment et peaufinée par le temps, scrutant une multitude d'objets comme la ville, les jardins publics, les sports, et de n'en parler alors qu'avec incomplétude, voire en introduisant des distorsions possibles ou des incompréhensions probables qui sont autant de trahisons envers une conception qu'il s'agit cependant de faire goûter; celui plus essentiel peut-être, de ne produire qu'un métalangage rationalisé, ordonné pour la clarté de l'exposé, alors que le style de pensée et d'écriture de l'auteur entraîne le lecteur vers les rêveries, les imaginaires, les lieux et les émotions de l'enfance ou du sport, vers les dimensions sacrées de la vie quotidienne, et l'analyse risque bien ici de pétrifier les sentiments et l'inexprimable, par une convention langagière injuste envers l'authenticité de la parole première de l'auteur. Entre ces deux attitudes, celle de l'attachement discret et secret à une oeuvre et celle $\mathrm{du}$ désir de la communiquer, la seconde est préférée ici tout en ayant conscience du risque de faire perdre une qualité émotionnelle en l'objectivant, de transgresser une pensée originale en produisant un langage technique, de refroidir par l'analyse des conceptions buissonnières situées hors des chemins balisées, de réduire la complexité en quelques fragments ordonnés dans lesquels l'auteur pourrait ne pas se reconnaître.

3 Il est une autre limite qui sera franchie, celle qui consiste à centrer cette note essentiellement sur la manière dont l'auteur perçoit le sport, alors que les objets auxquels il se réfère sont multiples, attitude qui peut prêter à la critique à cause de son réductionnisme. Cependant cette attention prioritaire accordée aux sports n'interdira pas de faire référence à d'autres objets premiers de l'oeuvre, comme la ville par exemple, sans qu'ils soient développés. Le choix du sport se comprend évidemment par notre position institutionnelle, notre appartenance aux S.T.A.P.S (Sciences et Techniques des Activités Physiques et Sportives), et notre curiosité se porte alors "naturellement" vers toute production concernant ce champ, mais il se justifie aussi pour d'autres raisons majeures. D'abord parce que l'hypothèse peut être avancée selon laquelle le sport peut paraitre ici une sorte d'idéal type au sens où en concentrant l'analyse sur lui, il est possible de mieux comprendre un ensemble de propositions théoriques et méthodologiques généralisables aux autres objets de recherche de l'auteur, ensuite parce que le sport n'occupe pas dans ses publications une place accessoire mais il est au contraire pleinement inclus dans une ensemble d'autres objets, enfin parce que la théorisation sociologique de Pierre Sansot permet de renverser bon nombre d'idées dépendantes de théories qui deviennent trop souvent des prêt-à-penser concernant les rapports entre le sport et la société. Se centrer sur l'oeuvre de Pierre Sansot, c'est encore ouvrir des perspectives de recherches inédites dans le champ anthropo-sociologique du sport dont les directions variées passionnent bon nombre de chercheurs attentifs au symbolique et à l'imaginaire dans la société, de telle sorte qu'il est capital de voir comment il peut se situer parmi ceux qui partagent des orientations de recherches en sciences sociales sensibles aux approches herméneutiques, phénoménologiques ou poétiques, loin d'être consensuelles. 
Il existe encore une dernière réserve qui témoigne du caractère partiel, voire partial, d'une telle présentation. Elle tient au parti pris de privilégier des axes synchroniques, transversaux à une pensée dont il faudrait aussi souligner la construction diachronique. En effet, ces travaux se distribuent sur une vingtaine d'années et s'inscrivent dans le temps. Cette dimension ne devrait pas être négligée car elle permet de prendre en compte la production en sciences sociales de cette période dont il est possible de penser qu'elle contribue à l'édification de la pensée de l'auteur de deux manières : soit en confortant ses thèses, soit en s'y opposant, deux manières différentes qui peuvent concourir cependant à l'amplification progressive d'une pensée. Tenter alors de rechercher le sens d'un travail, c'est certes extraire le "noyau dur" originel qui en marque sa spécificité, mais c'est rendre compte aussi, de manière obligatoirement incomplète, des attaches et des distanciations théoriques qui ont permis l'autonomisation d'une pensée dans un champ très surveillé. C'est là une façon d'entrer dans des problèmes de construction de la réalité sociale, dans les conflictualités d'une "intelligence du social" (Berthelot J-M., 1990). Ainsi, il faudra montrer comment une pensée du social se construit ici par des sédimentations anciennes et par des considérations puisées dans les productions sociologiques contemporaines venant parfaire une conception qui ne cesse paradoxalement de se complexifier tout en paraissant de plus en plus dépouillée au fil des ouvrages, ceci semble-t-il, afin de donner toute leur fraîcheur aux observations que les théorisations précédentes risquaient de masquer. Diachronie et synchronie de la pensée doivent donc paraitre ici étroitement connectées malgré le choix d'une présentation qui en privilégiant des thèmes, pourrait paraître relever davantage de la seconde perspective.

5 Malgré toutes ces réserves liées à l'excès d'ambition de cette note (une production collective consacrée à l'auteur sous forme d'hommage est annoncée aux Cahiers de l'Imaginaire), il s'agit ici seulement de transmettre un peu de la fascination qu'exercent les conceptions de Pierre Sansot, celle-là même qu'il voue aux sports et plus particulièrement à ceux qui comme le rugby ne valent que parce qu'ils conservent une grande part d'authenticité dans la dérive moderniste. Les orthodoxes de la méthode sociologique se risqueront à quelque critique de notre démarche : la proximité affective de l'objet ne risque-t-elle pas de perturber l'interprétation objective d'une oeuvre? Mais n'est-il pas admis aussi que l'objectivité n'est qu'un fantasme scientifique dont il faut se débarrasser, sans pour autant renoncer à la logique de la preuve? Dans ce sens, il est difficile de ne pas présenter une sociologie inclassable, séduisante, déroutante, voire subversive, attentive à saisir les objets et pratiques ordinaires de la vie quotidienne au moyen d'une approche "poétique" rompant avec la scientificité froide et standardisée, caractérisée par une réflexion théorique armée de solides références aux courants philosophiques et anthropo-sociologiques - dont la présence est souvent discrète-, laissant apparaître des ruptures par rapport à d'autres modèles d'interrogations du social et procédant à de nouvelles constructions de la réalité sociale jaillies d'une observation ethnologique fulgurante, turbulente et toujours maîtrisée. Et puis pourquoi bouder malgré toutes les prudences énoncées une approche du sport réalisée par un de ces rares universitaires qui malgré l'autorité accordée par leurs pairs, ose donner toute leur valeur à ces pratiques ordinaires en leur consacrant des pages passionnées qui n'ôtent en rien à la réflexion argumentée, voire critique ? C'est là aussi un signe qu'il existe bien une sociologie impliquante capable de faire surgir une connaissance novatrice à partir de cette harmonie entre la subjectivité et l'objectivité, 
une approche que Pierre Sansot qualifie d'"objectale" (1973: 9 sq.), l'objectivité étant réductrice.

6 Ces quelques remarques préliminaires pourraient paraître académiques au lecteur pressé d'arriver aux faits, mais elles sont justifiées tant le caractère réducteur de cette note est évident. Les objectifs ne peuvent être que limités : viser à faire découvrir/ redécouvrir la présence d'une "sociologie poétique" du sport, parce que comme tout fragment de la vie sociale, le sport comme phénomène collectif est irréductible à toute vue univoque (1986 a : 7), il se donne à lire dans des paroles, à voir et à imaginer dans des symboles qui transcendent le réel tout en lui étant lié.

7 Pour cela, de manière classique, à partir d'une bibliographie non exhaustive, quelques directions -clé seront dégagées pour cerner la théorie du social de l'auteur, en usant pour cela tantôt du style indirect, tantôt de citations permettant de sentir le phrasé de son écriture et de rester près d'une pensée même si elle est reconstruite pour les besoins de l'analyse. Le sport servira ensuite d'étude de cas à l'opérationnalisation de l'approche théorique qui sera illustrée par des entrées reconstituées autour de thèmes estimés majeurs dans l'oeuvre. Enfin, de manière plus libre et plus risquée, la singularité des thèses de Pierre Sansot sera envisagée en les insérant dans le champ des sciences sociales, notamment en les comparant à d'autres théories, proches ou distantes, mais en complexifiant leurs apparentes oppositions ou concordances, en entrant donc dans le débat sur les constructions du social régi par des antagonismes apparemment irréductibles.

8 L'objectif sous jacent à cette note vise aussi à démontrer comment la sociologie du sport qui se construit sur des paradigmes issus des grandes sociologies contemporaines, gagnerait à intégrer dans ses programmes de recherche une approche qui se distancie d'un scientisme froid et qui prive l'homme de ses capacités imaginales empêchant l'observation de la créativité sociale. Il faut alors voyager dans les lectures de Pierre Sansot qui invitent à nous "humilier devant le sensible" (1986 a : 211) et voir dans le sport ce que Mikel Dufrenne disait à propos de ses analyses de la ville, aller "Plus bas, vers cette co-naissance de l'homme et du monde", vers la découverte d'une "vérité première, une vérité familière que pourtant nous vivons sans nous en rendre compte" (Préface, $1973:$ 4).

Une pensée interprétative

Qu'est-ce qui fait l'originalité d'une approche sinon le "travail" des concepts ou des notions au sens où l'entendait Georges Ganguilhem, c'est-à-dire leur approfondissement permanent, leur précision progressive, leur variation dans les formes, leur capacité à révéler des traits inédits de la réalité sociale, de telle sorte qu'elle parvient à être reconnue dans un champ voire même à faire école. Le terme de "pensée interprétative" (1986 b) sera majoré ici pour condenser en un trait un ensemble de caractéristiques présentes sous des expositions variables dans l'ensemble des publications de l'auteur. Elle seule peut exprimer le "sensible" en reconnaissant toute la puissance de l'imaginaire, tout ce que notre "société imagine" (1986 a : 206 ), car l'imaginaire apparaît bien comme cette faculté "d'outrepasser le réel", d'aller "audelà de ce qui est affecté par le sensible" (1993 b: 411). L'imaginaire vaut par sa capacité d'inventivité de notre monde dont le dévoilement ne peut se produire qu'au moyen d'une approche poétique irréductible à une appréhension conceptuelle et scientiste.

L'interprétation 
"Interpréter, décrire, réciter", sont des termes majeurs pour "sauver le sensible" (1986 a :11). Ils constituent de véritables pivots autour desquels se projettent les regards de Pierre Sansot sur la réalité sociale et s'organisent moins en une méthode qu'en une approche. La différence entre ces deux modes d'appréhension est importante : si la première génère des attentes prometteuses et nécessite une certaine technicisation même si elle prétend ne jamais être une technologie, le terme de "pensée", plus modeste, paraît plus ouvert et laisse plus de liberté à l'observation du social en évitant l'enfermement et en autorisant des perspectives moins limitées (1986 b). Concevoir ce type d'approche spécifique, c'est postuler au plan épistémologique que les instruments servant à appréhender le social sont des "théories matérialisées", des "grilles révisables" et que "l'interprétation intervient à tous les niveaux de la recherche scientifique" (1986 b : 39), tout simplement parce que l'homme, dans le cadre de la vie quotidienne, est un extraordinaire producteur de signes dans son langage, dans ses actes, dans son existence, dans ses capacités inventives, dans sa conception de projets etc., signes ambigus par leur "excès de richesse" dont le sens est difficilement saisissable : les faits et les comportements qui ne signifient rien comme tels doivent aussi être constitués.

11 Mais le problème est de légitimer un style de recherche dont il est postulé qu'elle peut révéler par la description, des "nappes de sens, exhiber toutes sortes de signes sans chercher à les formaliser dans une théorie englobante et sans verser dans l'impressionnisme" (1986 a : 6), recherche irréductible en outre à toute autre approche. En tout cas, elle ne peut s'accorder avec une pensée qui appréhenderait les comportements humains dans une perspective "exclusivement mécanique" (1986 b : 40), dans du mesurable, dans la statistique, car si l'utilité d'une telle voie est admise, elle est inappropriée ici, car sens et interprétation disparaitraient alors.

Un tel choix liant théorisation et opérationnalisation se manifeste dans les prudences de l'auteur à saisir les comportements sportifs dans une seule dimension d'efficacité (1988: 269 ), ou par des résultats qui certes conservent toute son admiration quant à leur rigueur, mais dont il craint qu'ils n'aboutissent à une "certaine déréalisation, à la tristesse d'un monde où l'homme serait absent" (1988: 276). L'anthropologie de Pierre Sansot s'attache à échapper aux causalismes et à toute réduction de la totalité sociale et s'ancre dans des lieux contribuant à maximiser la dimension expressive des productions humaines : la parole, le corps et la gestuelle de l'autre. Ceci s'accompagne d'une conscience des difficultés de cette pensée interprétative puisqu'elle ne fait que redoubler une interprétation première et spontanée, produite déjà d'une symbolisation de l'univers matériel ou idéologique d'une culture, mais qui ne constitue en aucun cas un obstacle puisque existe un en-soi de la réalité dont il convient de ne pas s'éloigner, même s'il est difficile à atteindre (1986 b : 40).

La parole

13 Son importance est déjà présente dans la publication de sa thèse (1973) et ne cesse d'occuper l'espace méthodologique des analyses ultérieures. Mais de quelle parole s'agit-il ? Quel travail théorique l'auteur opère-t-il pour lui conférer une légitimité scientifique alors qu'elle peut être facilement l'objet de critique ? La sociologie ne nous apprend-elle pas à nous en méfier, à détecter ce qu'elle cache et à n'accorder aucune confiance au vécu, à l'affectivité ou à la subjectivité qu'elle exprime au nom de la conscience aliénée qu'elle ne fait que traduire? Alors les objections risquent de se manifester au regard d'une approche qui entend étudier les réalités sociales telles 
qu'elles se présentent, "souvent en usant du langage qui sert à les désigner" (1973: 10), réalités qui peuvent être la ville ou les sports, et que le commun exprime par des mots qui fondent toute la valeur de l'objet, contrairement à la pensée scientifique, "nocive" et "inexacte" qui les déréalise (ibidem: 11). Concernant la ville, la différence par exemple est grande entre le langage neutre du technocrate qui désigne la rue par un terme noble "l'artère", ou les jardins publics par les "espaces verts"(1993 a ), et qui en même temps élimine l'homme de la relation affective qu'il entretient avec elle, "Décrocher de ce langage, ce serait passer à un autre plan qui oublierait la relation affective des hommes et des lieux, ignorer la relation primordiale qui vaut selon nous, la peine d'être étudiée" (1973: 11). Notre imaginaire "ne possède pas un pouvoir d'idéalité (de négation du réel); il n'est pas pour autant englué dans le monde. Il le redouble " (1993 b : 413). D'où l'importance donnée par l'auteur à la restitution de la parole quotidienne "prise dans le cours ordinaire de nos pratiques, de nos préférences " (ibidem : 413).

Pour appréhender le sport, le rugby en particulier, lieu privilégié de la parole parce qu'elle participe au défi jeté à l'adversaire (1988: 268), Pierre Sansot sera très attentif à saisir l'authenticité du langage et à détecter les risques des stéréotypes qu'il peut véhiculer. Le débat portera alors non pas sur la linguistique ou sur les variations du langage selon les catégories socioprofessionnelles, mais se situera dans une dimension "éthique, voire ontologique" (1986 a: 48). Le langage permet en effet la mise en évidence de l'affinité des hommes avec leurs pratiques et quand il est familier, il est comme un poème, aussi polyphonique et polysémique. Pour l'entendre il doit être interprété et non seulement décodé (1986 b : 41), seule voie pour que la ville, les sports ou tout autre objet se parent du sensible.

Bien plus, cette autorité donnée à la parole comme dévoilement du caché social s'accompagne du recours au "vécu, au romanesque, aux effusions subjectives" (1986 a : 59 ) et privilégie l'émotion qu'elle peut signifier. Le sport, la ville ne peuvent être révélés que par cet artifice, cette ruse, sans pour autant croire que leur "coeur" peut être atteint par cette voie. Tout simplement, l'exploration de l'objet ne peut révéler plus que ce que l'approche autorise et il est vain de penser qu'un "en-soi" dans sa vérité puisse être appréhendé. L'auteur fixe par là les limites de l'interprétation, mais ose pour cela aller au plus profond de cette approche en magnifiant la parole, en donnant tout leur poids aux anecdotes, en signifiant l'alliance entre le "patent" et le "latent" dont la force dynamique est impressionnante, en montrant comment s'y précipitent non pas les "motivations" mais une mémoire donnant toute consistance à l'objet parce que s'y déploient rêveries imaginaires et souvenirs, c'est-à-dire un espace affectif obscur irréductible au langage technocratique des urbanistes ou rationalisant des professionnels du sport (entraîneurs, professeurs), ou à toute formalisation d'une pratique spontanée.

16 Ainsi s'affirme la nécessité d'une interprétation poétique, conséquence exigée par la nature expressive (1986 b : 41), attentive à repérer les métaphores, "fête des mots" (1986 a : 46) jointe à la nécessité de reconnaître certes un "métalangage" - celui-là même que l'ethnologue utilise comme exigence de connaissance liée aux règles et limites de l'approche, à une épistémologie en acte puisque le monde ne répond pas s'il n'est pas questionné (ibidem : 46) - mais à l'égard duquel Pierre Sansot veut garder des distances, puisqu'ici encore, cela risquerait de le priver d'une liberté qu'il scrute et qu'il veut restituer dans la vie sociale, liberté saisissable qu'au prix d'une certaine 
désinvolture jamais distante d'un engagement (ibidem: 47). En effet, ce métalangage pourrait bien nous "exiler du sensible social" (ibidem : 73) et exténuer l'émotion de la parole, masquer la "joie de redoubler les choses, de métamorphoser les légendes, en un dire cocasse ou sublime ou attendrissant, de les volatiliser pour rien, en mots" (ibidem : 48). C'est cette parole créatrice que guette l'anthropologie de Pierre Sansot, tout en n'étant pas docile à son égard, mais en privilégiant la "re-création mimétique à la reconstruction" (ibidem: 49), toute prête à signifier une "forme extrême de l'imaginaire" (1991 b : 73), parce qu'elle peut se libérer de tout "différent". Alors, pour apprécier la ville ou le rugby, pour appréhender "leurs parures sensibles", il suffira d'évoquer quelques "noms de rues aimés et connus" (1986 a : 50), ou quelques noms de joueurs prestigieux qui constituent la légende du rugby (1990 a), et de se dessaisir de tout commentaire qui se laisse aller à des effets de style, qu'il vienne des journalistes ou des "professionnels des beaux discours" (1986 a : 72-73). C'est à cette condition que le langage peut "charger de sens les cérémonies sportives", en s'ancrant à la réalité de la pratique, ce langage populaire saisissable dans les vestiaires, pendant ou après le match, celui de la mêlée qui sort des "tripes", celui qui s'inscrit dans une tradition vivante (1986 a : 73) loin de l'idéologie dominante, celui qui reste irréductible à toute fonction idéologique qui "rationalise, multiplie les démonstrations, rabâche pour mieux convaincre" (1993 b : 412), celui qui laisse vibrer l'affect et l'émotion et qui n'abolit pas les rêveries collectives (1991 b : 74), celui qui s'ouvre à un "imaginaire collectif" (ibidem : 73), qui restitue une "socialité effervescente au-delà du coeur de l'institution, de ses usages et rites déterminés" (1988:270) et qui ne servira jamais à promouvoir une politique ou un ordre social, quel qu'il soit" (1993 b : 412).

Pour en saisir le sens par la pensée interprétative, Pierre Sansot reconnaît le caractère exemplaire de la pratique psychanalytique, notamment par le rôle du latent, en demeurant soucieux de l'engagement herméneutique, en ne masquant pas la présence $\mathrm{du}$ contre-transfert sans cependant relever l'hypothèse de la présence de forces obscures puisqu'il ne s'agit que d'observer. La pensée interprétative se trouve au croisement de plusieurs disciplines et apparait "comme la voie supérieure qu'emprunte une culture pour de comprendre à travers les signes qu'elle produit" (1986:42).

Outre la parole qui est d'abord "une voix qui nous heurte avant d'être un message que nous décodons" (1991 a : 34), Pierre Sansot accorde autant d'importance au corps et la gestuelle.

Le corps, la gestuelle.

19 Convoquer le sensible ou restituer ce que les sens perçoivent, implique une attention aux attitudes du corps, aux postures, aux relations que les hommes entretiennent pour jouer, séduire, affirmer un pouvoir (1986 a: 12). L'évocation des plaisirs ou des malheurs de la culture populaire par exemple, s'opère en restituant l'émotion qui touche l'observateur. Le corps ici doit être révélé au moyen du sensible perçu comme élément premier de l'enquête et non comme forme déterminée par une approche analytique mêlant cette donnée à une théorie préétablie la privant alors de tout ce qu'il peut évoquer. De même que la pensée interprétative ne peut s'accommoder d'un langage rationalisant bloquant toute émergence de l'affectif, la lecture du sensible corporel ne peut être réalisée non plus par du mesurable : notre espace, notre rapport au monde "se joue selon des modalités plus flexibles, confuses à dessein..." (1986 a : 13).

Ici encore, cette approche même ouverte nécessite une intervention méthodologique scientifique qui s'opère sous la forme d'une mise en ordre de la perception, et 
l'interprétation, toujours nécessaire, se réalise sans qu'une coupure s'établisse entre le savoir et le perçu. Loin de tempérer les signes du corps, Pierre Sansot pour magnifier le social leur donne toute leur ampleur en étant attentif à l'importance des rituels. Dans une approche toute goffmanienne, son oeuvre fourmille de matériaux nés de l'apport d'enquêtes solides et d'observations personnelles minuscules permettant de rompre avec la rigidité des cadres sociologiques préétablis et avec une microsociologie perturbant la généralisation des observations (1991 a). Ce sont là des conditions pour lire la pluralité du social, même si cette hétérogénéité méthodologique peut paraître peu orthodoxe au regard du standard de la recherche. Il se distancie cependant d'Erving Goffman qui selon lui trahit cette vie sociale en n'ayant pas su échapper au langage formalisé (1986 a : 19).

21 C'est à la description de la culture populaire, "lieu d'élection du sensible" (1986 a : 20), à laquelle va s'attacher Pierre Sansot dans une approche rompant avec certains travaux de la sociologie figurative, trop centrés sur une théorie du langage ou sur une forme qui fige la richesse de son expression dont le corps constitue aussi un excellent analyseur. Ainsi préfère-t-il à cet "étrange Pierre Bourdieu", Richard Hoggart dans La culture du pauvre (1970), car ses descriptions montrent comment "un tel milieu se régale de tout ce qui affecte les sens (1986 a: 20), en excluant le métalangage, les statistiques, l'emphase méthodologique et en livrant des expressions verbales et corporelles non dévoyées par la glose scientiste. La culture populaire fait l'objet de diverses acceptions mais Pierre Sansot dans un souci de se distancier des écoles la liant aux groupes sociaux, aux classes sociales ou au prolétariat, entend restituer les pratiques ordinaires des "gens de peu", les modes de vie et les pratiques culturelles de ces personnes modestes qui rassemblent les individus dont "les destins peuvent diverger" (1991 a : 14). Ainsi une priorité est accordée à la description de la vie quotidienne, à ses plaisirs et à ses excès, en s'attardant soit sur les lieux (les rues, les stades, la maison, les trottoirs, le terrain de boules, le camping, les bals, les parcours du Tour de France, les squares, les parcs), soit sur les objets (le tricot, les casseroles de la ménagère, les signes ostentatoires, le pliant, les plats, les objets du camping, le ballon de rugby, les boules), soit encore sur les postures (les rituels et la théâtralité des jeux de boules, la magie du pointage, l'acte du guérisseur - et non les "médecines parallèles" -, les scènes de ménage, les techniques de contrôle du ballon de rugby ou de football, la confrontation à l'occasion de défis de force et d'adresse dans les courses à vélo ou au mât de cocagne), bref à ce qui paraît relever souvent d'un caractère festif et luxuriant, à ce qui donne sens et saveur à ces moments de la vie sociale et qui conduisent les hommes à une autonomie relative loin de la dialectique égalité-distinction et d'une influence de la culture dominante sur la culture dominée, et qui les rend capables de bien plus, notamment "de réinventer, selon leur bon plaisir, leur manière de vivre, en toute candeur et sans se soucier du reste de la société" (1991 a : 215).

Alors les mises en jeu du corps populaire sont repérées dans les sports et prennent des directions différentes. D'abord par le choix que l'auteur opère dans une multitude d'activités sportives possibles en privilégiant le rugby (1990a), "le football des trottoirs" et le jeu de boules (1991 a), le Tour de France (1986 a), c'est-à-dire des pratiques où se reconnaît encore un caractère festif non perverti par la modernité des nouveaux sports; ensuite dans la symbolique qu'ils expriment à partir des représentations du corps, notamment par l'appel à l'imaginaire, "Le corps humain quand il vibre est à la fois du côté du signifiant et du signifié et il constitue une extraordinaire matrice de sens "(1986 a : 70). Alors, la mêlée et la touche au rugby ne 
valent, la première que par le contact avec la terre ou la boue, la seconde que par les joueurs qui s'élèvent dans le ciel pour capter la balle "presque à hauteur des nuages" (ibidem: 71), offrant "une belle alternance des gestes et des éléments, de l'humain confronté à la glaise originelle puis à l'immatérialité des airs..." (ibidem: 71). Intelligence du corps encore et symbolique élargie à la perfection technique du drop, aux automatismes qui "mettent entre parenthèses notre existence individuelle et collective (...). Chaque fois que le corps exulte, le nôtre, ou d'autres corps avec lesquels nous entrons en sympathie, nous ne cessons de donner un plein sens à ce que nous voyons s'engendrer devant nous" (ibidem: 71). Contre les lamentations du dressage sportif, l'attention se fixe sur "la façon dont le sportif appréhende et réinvente son corps et l'espace-temps dans lequel il évolue" (1991: 73). La symbolique révélée par le corps sportif ne vaut pour Pierre Sansot que parce qu'elle renvoie à l'imaginaire.

Le sensible et l'imaginaire

Accéder au sensible, c'est rendre compte de "résidu significatif" (1986 a : 35) que beaucoup de recherches ethnologiques ne peuvent résorber puisqu'elles dépendent d'un parti pris méthodologique qui les régissent, même si Pierre Sansot voit en elles une source capitale d'information sur le sensible car l'enquête ethnologique "est écoute, retentissement et accueil à la parole des uns et des autres" (ibidem : 114). La quête du sensible se lie à celles d'entités indéterminées, assez proches des abstractions les plus pâles (ibidem: 35), rapportées non pas à des objets précis, par exemple la description d'un club de rugby, mais au rugby en général dont la place s'apprécie par rapport aux sports collectifs ou par rapport aux sports plus récents.

Non désignée comme telle, la notion de sensible est cependant présente déjà dans son premier ouvrage publié dans la collection d'esthétique dirigée par Mikel Dufrenne, ce qui peut être considéré comme un indicateur du fond de sa thèse puisqu'il s'agissait pour Pierre Sansot dans Poétique de la ville (1973), d'affirmer que le monde possède un sens dont il s'efforce de récupérer les bribes à travers les portraits du clochard, du chauffeur de taxi ou de la Prostituée, sans neutraliser ni la ville ni l'homme dont l'existence ne peut être considérée dans sa finitude "tragiquement atone et arythmique", prêt au renoncement et à l'exil, mais dans "son appétit d'imaginer" (1973 : 410). Une toute puissance est accordée à l'imaginaire comme catalyseur de sens, "Si une ville nous touche, au point de la ressentir comme la chair de notre chair, au point de la réinventer au plus profond et au plus obscur de notre être (et c'est bien cela imaginer) c'est qu'elle vient à l'être dans une explosion de couleurs, de sons, d'odeurs et que nous devons constater sa générosité créatrice - laquelle nous contamine et exalte notre sentir" (1993 b : 412).

Cette quête insatiable de l'imaginaire et du sensible sera présente aussi dans Variations paysagères (1980) où le regard sur les banlieues ne s'abandonne pas à l'anonymat, dans La France sensible (1985), où la France est vue comme un fait d'imagination-sans ignorer le rôle de l'économie -, ouvrage dans lequel le terme apparaît dans le titre pour la première fois puis repris dans Les formes sensibles de la vie sociale (1986) où la notion est là théorisée et retravaillée à travers d'autres objets qui sont chers à l'auteur : la banlieue, les foules, ses déambulations imaginaires dans son quartier à Grenoble (son "fragment urbain").

26 Le sensible est défini par "ce qui nous affecte et retentit en nous" (1986 a : 38), "ce que les hommes ressentent" (ibidem: 115), "ce qui nous apparait et nous affecte" (1987: 269), ou encore le niveau où "se produit la conjonction la plus élémentaire et la plus 
énigmatique (la plus admirable) du sens des sens", sens compris comme "sphère de significations" jamais extraite des "empreintes" sensorielles (1986a:5) difficiles à cerner dans leur réalité puisque leur signification risque de s'appauvrir par une verbalisation obligée. Ici la disjonction sens-verbalisation est réfutée et veut rencontrer un code, une "expérience du monde" à l'origine de notre gestuel, de notre sensorialité en maximisant "ce qui nous éveille ou nous brusque ou nous fait chanceler ou nous attendrit" (ibidem : 6), sans tomber dans l'impressionnisme et sans disjoindre le réel et l'imaginaire (ibidem: 39), imaginaire qui "ne constitue pas une évasion hors du réel mais une autre façon d'être en relation avec lui" (1993 b : 411).

Sa sociologie figurative s'attache à la représentation d'objets dont l'approche sensible est révélée au moyen d'images provenant du "monde des choses et des objets vivants lorsque je les considère comme un ensemble de formes, de couleurs, de sons indépendamment de l'usage que je pourrais en faire et ceci par une sorte de sublimation esthétique que nous ne réservons pas à quelques uns", car les hommes dans leur vie quotidienne sont affectés par ce qui les touchent pour rien parfois, hypothèse qui laisse ouverte l'emprise de l'imaginaire (1986 a: 40). Sensible et imaginaire sont indissociables et appréhender le réel ne peut se faire que par des "conduites symboliques, circulaires", il ne peut se laisser posséder même si par exemple la perception de la ville lors d'une promenade suit une harmonie qui permet de l'identifier, précisément par l'image à la fois présente et invisible qui s'en dégage.

Comment illustrer alors le sensible? Peut-être en tentant de repérer quelques notions connexes qui paraissent capitales dans l'oeuvre comme celles de sacré, d'émotion, d'imaginaire, de légendaire en étant conscient du parti pris de l'auteur : le sensible est un choix pour mieux exposer la magnificence du réel.

Sacré, émotion et production symbolique

29 Les formes du sacré sont déjà présentes dans Poétique de la ville (1973: 54-63), où Pierre Sansot montre comment le sacré oriente la cité (la cité médiévale préoccupée d'un Dieu transcendant abolit la ville au sens où l'étroitesse des rues préoccupe peu, car cela est inessentiel au regard des valeurs les plus hautes, célestes), comment il se projette dans l'espace urbain (le plan d'une ville romaine n'est pas le produit du hasard et repose sur une division quadripartite et l'orientation de la ville se situe par rapport aux trois portes), comment les lieux sont chargés d'histoire, comment les poètes comme Gérard de Nerval ou Aragon ont saisi des lieux et les ont investi d'une symbolique très riche (tracé imprévisible qui évoque le labyrinthe et son enfermement, découverte sordide source d'enrichissement personnel, le tunnel comme passage et médiation obscure), ville à caresser et à pénétrer comme le corps féminin, "elle possède ses creux et ses points chauds (ibidem : 57). C'est cette voie que l'auteur va suivre pour dévoiler la ville, les lieux où se mêlent le visible et l'invisible vers lesquels l'homme quand "il ne croit plus en un Dieu transcendant, peut insensiblement tendre" (ibidem: 62). Il jette là les éléments du sacré pour décrire non seulement une ville "qui nous fait être" et non pas "d'une ville neutralisée, déréalisée, désubtantialisée" (ibidem : 61) mais aussi, plus tard, pour décrire le sport.

Concernant cet objet, le sacré peut être compris comme un terme générique couvrant les rituels, les calendriers, les moments d'émotion intense, le sensible, l'héroïsation de certains êtres (1990: 60). S'agissant du rugby, l'auteur ne craint pas les procédures d'amplification et rend hommage au "positif", au "superbe", à ses excès, pour le saisir dans sa "visibilité jubilatoire", pour restituer l'émotionnalité d'une rencontre - même si 
les études causales peuvent conclure à un épiphénomène - en prenant le risque que son approche puisse verser dans une poésie médiocre "sous couvert de rendre hommage à l'apparaître de la vie sociale" (1988: 269). De même que sa description des joueurs échappera aux catégorisations qui lient leurs manières de jouer à leurs appartenances de classes, telles que les entrevoit Christian Pociello dans l'ouvrage qu'il consacre au rugby et qu'il trouve par ailleurs remarquable (1983), pour montrer, par l'appui des rêveries bachelardiennes, les complémentarités entre les "sauteurs" et les "tracteurs" plutôt que leurs oppositions qui doivent être nuancée alors.

31 A propos du sport de manière plus générale, c'est l'analyse du "résidu" négligé par les analyses critiques qui est prioritaire ainsi que la volonté de hausser le sensible comme catégorie essentielle d'entendement parce que "les drapeaux, les chorales, les visages crispés par l'effort, les sursauts d'un stade, les gestes inspirés d'un individu ou d'une équipe, la chaleur des vestiaires, tout ce sensible auquel nous attachons une extrême importance et qui trouve sa gloire jusqu'à émouvoir les stades, jusqu'à bouleverser la physionomie d'une ville..." (1986 a: 63). Ici l'émotion captée se veut "authentique", "effervescente", "intense" et non pas régressive comme le décrit la sociologie critique (ibidem : 64), et les passions -conduites réactives comme la haine ou l'émerveillement -, livrées par le stade, dévoilent ce qui se passe sur la pelouse et à travers elles "une portion de terre se charge d'un supplément de sens" (1991 b : 71) produit dans l'ici et le maintenant. Ici encore l'imaginaire est bien le pivot produit par cette émotionnalité, dimension d'un sacré irréductible aux déterminations socioculturelles et se montre susceptible de posséder "un pouvoir instituant" (1991 b : 71). C'est ce "jeu d'émotions intenses" (1993 b : 180), comme "le plaisir, la douleur, la fatigue, la gloire, la mort, la déprime, la chute (souvent après la retraite)" qui sont décryptées dans les plus hauts faits sportifs parce qu'ils prêtent à rêver par l'admiration, la fascination, l'étonnement et dans lesquels plaisir et douleur sont mêlés dans les lieux "poétiques" où ils se déroulent.

En s'appuyant sur l'anthropologie de Marc Augé et celle de Georges Balandier (1974) qui montre comment en l'homme existent des dispositifs à symboliser, Pierre Sansot pense surtout "à une culture ou à des microcultures capables d'inventer leur propre symbolique à nulle autre pareille (...) : les contenus, les productions elles-mêmes ne se préforment ni se prédéterminent par des archétypes fondamentaux. Du sens advient là plutôt qu'ailleurs, à ce moment et non point à un autre, et, à vrai dire, nous serions bien en peine d'en avancer les motifs" (1986 a: 68-69). Or, dans le sport existe une capacité à inventer, une production symbolique lisible dans cette "intense émotionnalité", repérable dans le classicisme sociologique de Durkheim relativement à la fête ou à la religion, sans confondre les "communions révélatrices d'un ordre symbolique" et "les fusions quasi animales", une "communion dont la modalité sociale serait noble, élevée" et "une fusion quasi satanique et régressive" (1986 a: 75). La réponse est cependant difficile puisqu'une portée symbolique existe même dans les fêtes nazies et l'auteur propose d'abandonner le terme de communions mystiques ou celui de fusion plus caractéristique des assemblées politiques totalitaires, pour voir dans le spectacle "des élancements ", et chez les spectateurs "un entrecroisement des destins individuels " dans la mesure ou chacun, un moment, perd son identité pour devenir un être quelconque dans un ensemble qui le dépasse (1986a: 7). Mais cette identité peut être reconquise par une autre production symbolique qu'autorise le sport : celle "du sentiment d'appartenance à un club ou à une communauté", générée par l'effervescence d'une rencontre où s'affrontent souvent - par la destruction des 
emblèmes caractéristiques d'un club (souillure des drapeaux, destruction des totems) et par les médiations symboliques qu'elle provoque -, des valeurs, des rancoeurs, des nouveaux défis amplifiés par la mémoire collective (les contentieux) qui lient la ville à un club, dans une théâtralisation souvent destructrice parce qu'elle se rapporte au corps et à l'honneur, dans une tragédisation proche de celle du théâtre classique.

Sensible, sacré, émotion, imaginaire dans sa liaison avec le réel, s'exacerbent pour restituer des lieux, des pratiques, des hommes dont il importe de montrer les prolongements oniriques que le sport provoque en eux. C'est à ces "variations imaginaires" que procède Pierre Sansot, en nous invitant à rêver ce que serait une ville privée d'équipe ou qui descend dans une division inférieure (1986 a : 79).

Variations imaginaires

Ici encore, Pierre Sansot prolonge dans sa lecture du sport, les rêveries de la ville ou d'une région auxquelles il nous avait habituées $(1973,1980,1985)$, ou celles des lieux de l'enfance (1989 a). Dans sa théorisation de l'imaginaire, il paraît plus proche des conceptions de Gaston Bachelard que de celles de Gilbert Durand au sens où son projet ne vise pas à découvrir les structures anthropologiques de l'imaginaire (Durand G. , 1964, 1969, 1975), c'est-à-dire à "mettre en place les structures de l'imaginaire sportif" (1990 b : 64 ), mais à suivre plutôt Gaston Bachelard qui "nous a enseigné à rêver les éléments à partir des poètes et des écrivains : rêveries de l'eau, de la terre, de l'air, du feu" (ibidem: 63). Le sens que donne Pierre Sansot à l'imaginaire s'enracine dans la logique bachelardienne, imaginaire qui "présente l'intérêt de disposer d'un signe de rupture: la création (transfigurante et réductrice de l'objet au pur imaginaire) d'images" (1973 b : 413). Alors la perception des lieux ou du sport se réalise sur le mode de la rêverie, même dans les sports les plus violents comme le rugby. Ce sport lui apprend à vivre en "sympathie avec la Terre" (1990 b : 63) et si le joueur de rugby ne décolle pas de terre, "Les voltiges et surtout la circulation du ballon suffisent à donner l'illusion d'une suspension de nos lieux à la terre" (1990 a : 97). Quant aux joueurs, ils l'instituent "archéologue involontaire d'une préhistoire depuis longtemps disparue: des aurochs, des mammouths, des bisons, une race mi-divine, mi-animale, égarée au milieu des humains" (1990 a : 99 ; 1990 b : 64).

Les formes imaginaires sont exaltées et le sport se trouve magnifié. Pierre Sansot veut dégager la portée de cette admiration de l'exploit qui suscite l'émerveillement du stade et provoque les rêveries : désir de devenir le plus haut sauteur en hauteur du monde, risque de mort de l'alpiniste, martyr de la chair du marathonien, image du sublime provoquant l'émotion, quête de l'inaccessible et dramatisation de l'événement produisant ce mélange de réel et d'irréel ( $1990 \mathrm{~b}: 65$ sq.). Ce sont là des occasions qu'offre le sport de libérer les imaginaires d'où naissent une poésie et une capacité à créer de nouveaux symboles, à l'occasion par exemple du Tour de France, "Nous associons tellement cette course à la renaissance de la France que nous aurions souhaité qu'elle se gagne à Reims ; comme il aurait été beau que le maillot jaune eût été conquis définitivement là où les rois de notre pays recevaient l'onction sacrée ? Quel plus beau sacre pour un coureur que celui de Reims ?" (1991 b : 192). Ou encore rêveuse utopie évoquée par ce Tour de France dont la géographie se grefferait à la terre loin des grandes routes en une "coexistence ensoleillée du chrome, des tuniques bariolées, des muscles longiformes et d'un pigeonnier ou d'une ferme mal entretenue, quel bel hommage rendu à la France! " (1991 a : 194). Pierre Sansot retrouve dans le sport la présence du paysage dont il avait souligné l'importance dans Variations paysagères 
(1980), paysage qui "mêle l'observation du vécu et la représentation de l'imaginaire" (1992 a : 191), et dont la présence se manifeste lorsque l'homme devient un être sentant et que le monde s'appréhende par le sensible. Il entend s'écarter d'une définition traditionnelle du paysage pour le comprendre comme "un milieu qui tranche sur le reste du monde, dont les éléments vivent en connivence, qui imprime en nous certaines postures corporelles et une certaine forme de plaisir" (1992 a : 190). Dans ce sens, le milieu qui entoure le sportif guidé par une passion est partie prenante de la constitution de son moi, et une piscine, une salle de boxe ou des vestiaires ne sont pas des espaces exclusivement fonctionnels, comme Jacques Birouste l'avait aussi finement montré à propos de la nageuse et de son rapport à l'eau (1993).

Le sensible ne peut donc se révéler que par cet état d'esprit, cette capacité singulière à établir un rapport avec le monde et ne peut se circonscrire à une définition close enfermant toutes les potentialités et les expressivités d'un social dévoilées alors par cette approche qu'aucune autre ne peut rendre accessible. Le social se donne ici à lire, à sentir, à imaginer dans les expressivités corporelles, dans des formulations langagières serrées sur une authenticité et non dévoyées par un métalangage dont Pierre Sansot cherche à se distancier en sentant cependant le poids de la norme universitaire et la nécessité de faire science dans un champ très surveillé. Il rejette le scientisme qui refroidit -au nom d'une conceptualisation obligée et bornée -, tout ce qui fait la trame de ses observations : les moments chauds de la vie quotidienne rapportés à la toute puissance de l'image et des symboles. Pour cela, le fond théorique figuratif toujours articulé au réel sur lequel il s'appuie, entre en harmonie avec une écriture dont le style établit cette alliance difficile entre l'expression poétique et le regard concret sur les êtres et les choses. C'est là une approche susceptible de donner la preuve toujours liée à une "écriture émotionnelle" qui ne se veut pas fondamentalement littéraire, même si Pierre Sansot dit ne pas éprouver de honte au cas où il serait considéré comme un narrateur (1994 b: 65). Comment résoudre cette aporie née de cette double contrainte indissociable, le poids de la norme méthodologique pour faire science, et la restitution de formes sensibles avides d'espaces, de rêves et d'imaginaire par la poétique qu'elles suggèrent?

L'approche descriptive

C'est bien ce dilemme que Pierre Sansot s'efforce constamment de résoudre depuis ses premières publications. Comment faire science et restituer poétiquement la vie sociale? Comment établir une conjonction entre deux directions communément disjointes en sciences sociales sinon par une approche en adéquation avec la pensée interprétative et qui se fonde davantage sur l'observation que sur des a priori consistant à postuler l'existence de forces obscures régissant gestes, postures, paroles, modes de vie, bref les cultures? Comment concilier ses conceptions philosophiques avec le regard empirique qui se veut ordonnant quand il prétend être scientifique?

C'est à la révélation du latent, visée scientifique propre à l'approche clinique, à laquelle va s'attacher Pierre Sansot, sans rechercher les antécédents causalistes, mais en tenant compte des transformations de l'analyste au cours des entretiens. Ce sont ces fondements, croisant toutes sortes de disciplines qui servent de référents et de principes aux descriptions et qui donneront toute sa vérité à la quête du sensible en permettant aux objets révélés de s'ouvrir à certains "signes non perçus", "aux rumeurs de la socialité"(1986 a : 66 ), en entrant pour cela en intelligence avec eux, en révélant leurs nuances, en sacrifiant aux détails qui peuvent cependant porter une grande 
ambition, celle de dévoiler comme l'écrit Henri Leroux "les gestes majeurs de l'Anthropologie" (ibidem : 66).

Mais alors, quel sens faut-il donner aux propos de l'auteur quand il prétend ne pas faire "oeuvre d'ethnologue ou d'anthropologue. Nous cherchons à rendre hommage au sensible en tant qu'il est animé par la vie sociale" (1986 a : 99) tout en admettant cependant que "L'ethnologie demeure précieuse. Elle élargit notre horizon des possibles, elle aère notre existence singulière, elle donne à penser que nous pourrions vivre autrement" (ibidem : 100) ? Car c'est bien là, dans les relations de l'ethnologue à l'objet, dans le comparatisme qu'il établit entre ce que les cultures différenciées permettent d'imaginer et de symboliser, dans ce qu'il ouvre à un "univers plus donnant, plus riche, plus vibrant", que se conjuguent la richesse du terrain et les problématisations de la vie sociale, ce qui permet d'insérer la description du sensible dans le "mouvement de l'histoire" (ibidem : 33). Certes Pierre Sansot reconnait que son entreprise peut être contestable parce que le travail de l'ethnologue est toujours guidé par un intérêt théorique et que le recueil de données y est dirigé. Son orientation semble plus buissonnière et s'attache plus au pôle descriptif qu'à une théorisation excessive préalable bloquant les émergences, sans ignorer la tension liant les deux registres. Alors par exemple, dans Les formes sensibles de la vie sociale, le rugby n'est pas analysé par une méthode comparative visant à établir des différences entre deux villes ou entre les matches de la semaine et ceux du dimanche, mais l'auteur nous relate l'événement dans la durée, celle du dimanche, en décrivant ce qui se passe avant, pendant et après le match, ceci étant rendu possible grâce à une "longue pratique affectueuse" capable de restituer les émotions sportives (1986 a : 66).

Vis-à-vis du sport ou de tout autre objet, les "simples descriptions" sont préférées aux concepts ou "aux grandes thèses enrobées d'idéologie, même lorsqu'elles prétendent combattre et démasquer l'idéologie" (ibidem : 82), description que l'auteur veut "la plus pure, la plus éidétique possible" (ibidem: 113), sans que soit ignorée l'existence de déterminations socioculturelles. La quête de la production de sens enfoui dans un sensible se réalise donc dans une visée herméneutique par une méthodologie phénoménologique construite sur l'éloge de la description reposant sur différentes modalités d'enquêtes. En effet, aucune science ne peut avoir raison de l'apparaître d'une ville ou d'un corps, ni ne livrera toute leur vérité ou leur réalité. C'est là pour Pierre Sansot une preuve que la phénoménologie a toute sa place en nous apprenant que l'essentiel est dans un apparaître indépassable, ambigu et dans la perception que nous avons des êtres et des choses. Sa légitimité est affirmée alors puisque ces objets ne s'évanouissent pas sous le regard d'une science "objective" qui fait des hypothèses mais qui ne varie pas les approches. C'est "une voie difficile" (1973: 410) qui ramène à l'immédiat, et les "variations" de l'auteur appellent à la pluralité d'une perception, jamais unitaire ni béate. Le rapport entre l'observateur et la ville, le sport, ou les corps se fait plus riche, se fonde sur des images, des affects, tout comme les gens modestes retiennent moins le contenu de ce qu'ils voient ou de ce qu'ils entendent, que le côté spectaculaire s'offrant à eux et qu'ils retiennent : apparat, couleur, expression sévère des visages, c'est-à-dire le côté frivole. Un fait social est digne d'intérêt pour Pierre Sansot dans la mesure où il affecte ceux qui l'étudient tout en veillant à ce que le métalangage de l'observateur ne trahisse pas l'émotionnalité de la parole humaine. Plus qu'une méthode, l'auteur met en oeuvre "un tour de main ou plutôt un tour d'écriture" (1986 a : 52). 
41 Cette quête affectuelle permanente des formes sensibles, bien que liée à une phénoménologie, se réalise parfois sur le mode "classique" du métier de sociologue, c'est-à-dire sur des hypothèses de travail et sur des enquêtes de nature différentes (sur la ville, 1986 a ; les modes de vie des "gens de peu", 1991 a ; les sports, 1986 a, 1990 a , 1990 b) afin de parfaire ou confirmer cette connaissance de l'apparaître et faire naître cet "appétit d'imaginer" (1973: 410). Le passage qu'effectue Pierre Sansot du phénoménologique au poétique n'a rien de littéraire, contrairement à ce qu'un premier regard laisserait penser, mais se veut en prise avec le concret. L'amplification, la magnificence du social méthodologiquement construite, ne consiste pas en effet comme il le rappelle "à grossir indûment les phénomènes, à leur attribuer une importance qu'ils n'ont pas (nous verserions alors dans une vision du monde) mais à nous plier à leurs prétentions, à faire ressentir en eux ce à quoi ils aspirent et dont ils portent, en creux, la marque " (1973 : 413). La phénoménologie avec ses descriptions, s'oriente alors vers cette combinatoire difficile, celle du "réalisme poétique" (1973 : 414), parce qu'ancrées dans des lieux, dans l'inspiration des citadins ou dans le langage.

La ville et les stades retentissent dans les êtres, imaginent en nous et la rêverie évoquée n'a pas pour objectif, s'agissant de ces objets, de transformer le réel comme le font les critiques, mais "en prenant le parti de ce qu'elle invente du dedans", en montrant son caractère actif, en échappant à l'instrumental, "Elle s'empare des éléments, elle joue des éléments, elle joue des formes, plus encore qu'elle s'initie à leurs forces" (ibidem : 418). La description, au service d'une poétique de la ville ou du sport, en magnifiant le sensible, ne peut passer "pour la belle réconciliation symbolique qui cacherait les conflits qu'elle n'a pas la force de voir. Lorsque cette poésie sourd dans les cités et dans les actes des hommes, elle montre, dans un début de réconciliation que ceux-ci, dans les situations les plus terribles, n'ont jamais été écrasés sous le poids des fatalités sociales" (ibidem : 420). Il faut le comprendre, la description ici a statut scientifique. Sommée de donner la preuve, elle est au service d'une argumentation à laquelle n'échappe pas Pierre Sansot, consciemment. Elle s'articule sur un fond théorique dont la crédibilité est à démontrer vis-à-vis d'autres regards sociologiques concurrentiels portés sur les faits de la vie quotidienne ou de manière plus générale sur les cultures. Ceci sera abordé en dernière partie après avoir tenté de voir maintenant la perception qu'a Pierre Sansot des sports dans leurs liens avec le sensible et l'imaginaire.

Les sports, le sensible et l'imaginaire

Sans doute le rugby est-il parmi les sports celui que Pierre Sansot affectionne le plus à tel point qu'il est possible de dire qu'il est un analyseur des autres sports, dans la mesure où sa lecture sensible permet de mieux démontrer son positionnement original dans le champ des autres pratiques sportives, sans qu'il faille voir ici une analyse comparable à celle de Pierre Bourdieu. D'autre part, la pensée sensible de l'auteur se complexifie dans le temps et si une fidélité à la notion et aux approches qui avaient construit son regard sur la ville se retrouve dans les premières publications sur le rugby (1986 a, 1988, 1990 a), elle s'amplifie plus tard par la dimension du légendaire qui nourrit l'imaginaire et par la référence à d'autres sports qui résistent au temps comme le Tour de France. Ceci lui permet de redonner tout leur poids aux notions constitutives de son approche poétique du sport, le sacré, la fête, l'imaginaire collectif, les rêveries bachelardiennes, la dimension rituélique du spectacle, notions qui permettent d'envisager les sports non pas une dimension fixiste et immuable, mais dans leur aspect génératif, car les sports inscrits dans la tradition se transforment sous l'influence de la 
modernité tout en résistant au risque d'uniformisation et à la montée des sports narcissiques. La lecture poétique ou sensible ne se veut nullement nostalgique, mais réaliste, elle s'articule toujours avec le concret et les transformations sont dévoilées par une approche de terrain construite ici aussi sur une observation participante "objectale". Ainsi le joueur de rugby Jérôme Gallien notait la "discrétion étonnante" de Pierre Sansot lorsqu'il recueillait les impressions des joueurs qui l'avaient appelé "le Prof", ou quand il relevait leurs commentaires après le match en "buvant les paroles de Daniel Herrero (1990 a : Préface). La méthode privilégie la parole ouverte qui charge de sens le sport (Pierre Sansot comparant la parole de Daniel Herrero à celle d'un chef Guayaki parce qu'il fait entendre le chant de l'amitié et rêver les hommes en utilisant les mots qui sont plus ceux d'un poète que ceux d'un technicien, $1990 \mathrm{a}: 75$ ), en tenant compte des anecdotes exprimant de l'émotion (respect de Daniel Herrero encore qui lors des rencontres décisives porte une cravate, ibidem: 19, ou parole de l'arbitre rencontré par hasard qui lui par de ses qualités d'arbitrage car il a fait un sans faute malgré les difficultés à ne pas favoriser l'équipe locale). La méthode fait appel aussi à des descriptions mêlées de métaphores: "cérémonie" du match produisant du sens, avant-match de rugby qui établit une rupture avec la durée du spectacle, moment où le stade, masse de béton, s'éveille et "évoque la partance des bateaux ivres" et devient le lieu de rêveries ou de mémoire collective, lieu où les supporters se jettent des défis emblématiques, souffle suspendu des spectateurs quand les joueurs pénètrent sur le terrain; enchevêtrement des sentiments pendant le match et intense émotionnalité liée à la souffrance (1986 a 82 sq.), affrontement des hommes dans les "corps-à-corps émouvants" d'une mêlée, sorte de "chaos originel" (1990 a: 48). Toutes ces données sont recueillies et interprétées sans céder à la vision angélique des rencontres admirables qui comportent aussi une violence constitutive dans laquelle la force des corps est mise au service de l'intelligence (ibidem : 53-59).

Quelle vision Pierre Sansot donne-t-il alors du sacré et du légendaire, dimensions nourrissant l'imaginaire dans les sports ? Car la modernité, même dans le rugby, tend à techniciser la gestuelle, à rationaliser l'entraînement et avec le recrutement à modeler plus harmonieusement les corps et à faire disparaître les "êtres de légendes, les héros des combats homériques" (1990 a : 172) pourtant présents à travers quelques figures, démontrant ainsi la survivance de traditions dans la contemporanéité.

L'entrée dans le sacré

Pierre Sansot va prendre à contre-pied, la réfutation des approches durkheimiennes faite par Alain Ehrenberg (1988) et qu'il interprète ainsi : "non seulement le sport ne perpétuerait pas une nouvelle forme de sacré mais, pour prendre toute sa place, il lui fallait profiter de l'espace devenu vacant d'une religion défunte" (1990 b: 74), il ne reste plus à l'individu qu'à devenir performant puisqu'il ne sait plus où il va! Contrairement à ces thèses dont il reconnaît par ailleurs le "brio", mais qui ne valent pour lui que pour les lecteurs "si mode" d'Actuel ou de Globe, "qui ont choisi une existence lisse, en deçà de toute réflexion et hors de portée du peuple" (ibidem), Pierre Sansot va affirmer l'existence d'une permanence de valeurs dans le sport, qui n'ont rien de désuet notamment quand elles reposent sur l'accord des pratiquants "au vent, à la boue, au soleil" ou sur le bonheur d'être ensemble, alors si le sport "ne mérite pas d'être traité comme une religion. Il ne constitue pas, pour autant, une pratique née de nos désillusions" (ibidem). 
46 A certains égards cependant, dans une logique durkheimienne, il esquisse des rapprochements entre le sport et la religion, surtout par l'engagement du "pratiquant" (terme qui désigne autant l'adhésion au sport qu'à un culte), sans que les deux objets se confondent. Il admet ainsi que le rugby est "une "forme de religion" (1990 a : 9), qu'il constituait même à une époque "à la lettre une religion et où la passion qu'il provoquait s'apparentait à une recherche de l'Absolu" (ibidem: 29) au sens où il qualifiait l'existence, où il devenait un destin allant jusqu'au suicide pour le dirigeant qui n'accepte pas la défaite, où être rugby relevait d'une sorte de don. Ce caractère traditionnel qui lui reconnaît dépend d'une "forme de culture", d'une "philosophie", d'un art de vivre rompant avec le show organisé des spectacles devenant simples divertissements. Si le rugby suivait cette dérive il perdrait son caractère religieux (ibidem : 35) ainsi que son caractère moral que les entraîneurs s'efforcent encore de conserver. Par ailleurs les métaphores religieuses abondent pour donner sens au sacré, qu'il s'agisse des spectateurs ou des supporters qui sont les "officiants du culte" (1990 a : 66), lesquels quand les joueurs "quittent le stade les maillots humides (...) posent leurs mains sur ces saints suaires" (1986 b : 89), ou encore les stades qui font penser à d'immenses "cathédrales de béton" (1990 b : 66), ou bien les villes des clubs de rugby comme Toulouse, Brive, Béziers et Grenoble qui instituent une autre carte géographique et qui reviennent à nous de façon "liturgique, religieuse" (1990 a : 89), ou celles du Tour de France dont "les étapes ponctuent la ronde liturgique" (1991 a : 197), sans reproduire les formes d'un pèlerinage. La référence au religieux sert donc ici de méthode comparative analogique permettant de décrire le culte sans que ce soit une transposition simple de traits d'une religion au phénomène sportif. Le sacré décrit s'organise autour d'indices dont il reconnaît que les formes comme "l'émergence d'un calendrier et d'espaces spécifiques, de rituels strictement observés, de moments d'émotionnalité intense, de rassemblements communiels, de l'héroïsation de certains êtres" (1990 b : 60) peuvent convenir à d'autres manifestations du sacré, sacré qui peut être appréhendé dans trois dimensions reconstituées ici : l'émotion liée à la cérémonie, les rituels qui marquent son sens et la fête.

Si les émotions sportives participent à la mise en acte du sacré, c'est par leur intensité jaillie de l'événement vécu, qu'elles viennent de la clameur des spectateurs qui précède le début de la rencontre comme "une terreur du sacré, un effroi païen" lié à l'imprévisibilité de ce qui va se produire (1990 a : 62) ou bien des silences succédant aux exaltations, c'est-à-dire à cette oscillation de sentiments où alternent accablement, mélancolie et plaisir d'admirer "l'intelligence en acte" (ibidem : 64). Elles créent par conséquent une communauté, un rassemblement communiel mais non fusionnel dans lequel les individualités conservent leurs caractéristiques. C'est bien cette sensorialité auditive perceptible dans le stade qui fait la socialité ainsi que les comportements des joueurs et des entraîneurs soumis à une "part de magie, de pratiques incantatoires, de croyances obscures" (ibidem : 63) que décrit Pierre Sansot, ou encore les initiés du Tour de France assistant à cette cérémonie dans "une adoration unanime continue" qui ne se produira qu'un seul mois de l'été" (1991 a : 190). La sensorialité comme mode d'être au monde, comme modalité de connaissance du sport bien éloignée de la rationalité convenue, va ici prendre toute son importance.

Tout culte se fonde sur des rituels qui accompagnent l'événement sportif dont les liens avec le sacré doivent être établis. Ici, le comparatisme classique avec les sociétés traditionnelles, habituel en anthropologie pour traiter cette question n'apparaît pas 
pertinente. Comprendre les mythes et les rites en sport ne nécessite pas un recours aux références archaïques de sociétés autres, cela conduirait à l'envisager comme un rite de fondation, de telle sorte qu'à son insu, le sport permettrait de maintenir un monde sacralisé et plongerait dans une "tradition immémoriale" (1986 a : 67). Tout en louant les vertus heuristiques de ces thèses comparatistes, Pierre Sansot rompt avec elles pour montrer "comment l'actuel est destiné à se modifier, comment il se rejoue et se rethéâtralise en chaque circonstance" (ibidem : 69). Contrairement aux idées de Michel Raspaud (1983) qui interprète le sport dans sa liaison avec un sacrifice originaire, il considère que la rencontre sportive ne le réactualise pas, "puisque le résultat n'est pas donné à l'avance et que le sacrificateur devient parfois le sacrifié, puisque, en cas d'un match nul, rien ne serait joué et que la cérémonie deviendrait dérisoire (1986 a : 76).

Ici la lecture du sacrifice dans le sport qui "fait advenir le sacré et le rend crédible aux yeux des incroyants" (1990 b : 61), s'appuie sur les théories de Jean-Marie Brohm (1982) qui souligne la capacité d'engendrement de la mort réelle dans le sport, violence qui ne lui pas spécifique, mais que Pierre Sansot interprète dans une sensibilité à Georges Bataille (1967), comme "la part maudite de la condition humaine" (1990 b: 62), mais aussi violence "essentiellement symbolique; en quoi elle se rapproche des rites grâce auxquels les hommes d'une société exorcisent une violence plus originelle, sans pour autant perdre le goût de la compétition" (ibidem: 62). La fonction cathartique de la violence symbolique du sport se combine ici avec le réalisme d'une violence thanatologique réelle, pour révéler l'alliance entre la violence et le sacré et les capacités imaginaires qu'elle génère. Mais les risques de l'emprise de la violence aveugle sont toujours réels dès que la violence symbolique s'estompe comme en témoigne l'exemple du Heysel (1986 a: 101), et l'opposition violence symbolique à tendance cathartique et "la violence incontrôlée, non ritualisée est trop belle pour être vraie" (ibidem : 102). Pierre Sansot se demande si finalement "l'objet du sacrifice (de ce rituel) ne consiste pas en ce stade que l'on met à mort à la fin de chaque rencontre, que l'on abandonne au brouillard de la nuit, en un mot au désert du sens" (ibidem : 95).

La sacralité nous paraît alors devoir être lue dans un changement d'épistémologie accordant une toute puissance à cette foule qui fait le stade et réussit à faire "vibrer le béton" (1991 b: 72), épistémologie irréductible à l'analyse de la différenciation socioprofesssionnelle composant le public des tribunes et qui se centre plutôt sur les lieux transformant les paysages, sur la cérémonie qui se déroule dans l'instant dont l'imaginaire ne peut se confondre avec le spectaculaire, sur les rituels qui, comme l'a montré Christian Bromberger (1987), sont irréductibles à "une magie ignorante, de ce que la science expliquerait par la seule raison" (1991 b : 72).

51 L'anthropo-sociologie poétique de Pierre Sansot se concentre sur l'authenticité du rite afin que les jeux et les sports s'intègrent dans une quotidienneté non pervertie par la spectacularisation ou par la dérive des valeurs originelles qui les constituent. Elle s'attache à décrire des pratiques ordinaires comme le jeu de boules car la socialité chaude qu'il développe l'éloigne du sport de compétition moderne, artificiel et gigantesque, tout en montrant cependant comment ce jeu est accaparé par "une mythologie de la consommation" des touristes ou des vacanciers (1991 a: 157) inattentifs au respect auquel incitent les rites qui l'accompagnent. Ces pratiques peuvent servir de référence au football ou au tennis pour que ces sports cessent de "jouer en marge de notre existence quotidienne" (ibidem : 162) et l'amour que l'auteur porte le rugby ne le rend pas aveugle puisqu'il ne le reconnaît pas non plus comme une 
"culture" intégrée à la vie quotidienne (1990 a : 50). Cette même prudence, voire un "irrespect" s'exerce contre les pratiquants des nouveaux sports narcissiques - comme le monoski ou le surf des neiges - plus occupés à eux-mêmes qu'à la montagne, activités qui comme le rugby "se pliaient superbement à une instance cérémonielle faite de mythe et de rites", la montagne en effet avait précédé l'homme et avait suscité "monstres, titans et archanges" (1990 b : 70).

Dans cette anthropologie, la dimension rituélique du sport ne peut parvenir à fixer le sacré que si les rêveries et l'imaginaire auxquels il renvoie s'ancrent profondément à l'expression d'une mémoire ou d'une vie sociale intense et authentique, à la perpétuation d'une tradition culturelle soumise aux dangers du changement et ceci sans qu'il soit nécessaire de faire appel à des résurgences archaïques ou à la constance d'une forme archétypale (1986 a: 95). Outre ces formes du sacré constituées par l'émotionnalité des cérémonies et par les rituels, Pierre Sansot développe encore une autre forme possible d'expression du sacré, la fête.

53 La fête sportive à laquelle il fait référence n'est pas interprétée comme la classique transgression du rite, comme ce fut le cas des joueurs de rugby du Racing Club de France qui arborèrent sur le terrain "des noeuds papillon roses ou des maillots de bain façon avant-guerre" (1990a: 33), mais plutôt dans une tradition sociologique attribuant à la fête un caractère sacré se traduisant par "l'extase, l'exaltation, la dépossession, la transe, l'inversion des valeurs, la sortie de la vie quotidienne". S'accorder sur ce sens suppose l'adhésion émotionnelle au rituel dans lequel les individus s'expriment librement (ibidem : 34). Bien sûr, la fête transgressive sera décrite par ailleurs, à l'occasion de banquets qui donnent lieu à mille fantaisies dans les manières de table autour du pastis dans le Midi (1991 a: 103), ou lors de matches au cours desquels la farce et le défi par les parures emblématiques des foulards supporters sont omniprésents (1986 a : 88), mais son attachement à l'idée de sacralité dans la fête va aux rituels qui méritent le respect fondé sur une entente tacite entre le public et les joueurs, comme c'est le cas dans le jeu de boules où les réjouissances sont partagées grâce à la connaissance intime d'un code (fidélité du public, présence physique, parole chuchotée des joueurs ...), ouvrant à la rêverie et au mystère (l'adresse, la réduction de l'imprévisible, la ruse, l'accompagnement de la boule par la pensée et par le corps) (ibidem: 159 sq.), dans des rituels qui ne se confondent donc pas avec les rituels ludiques d'un public qui souvent occupe la scène au détriment des joueurs (1986 a: 88 ).

Encore une fois, la dimension sacrée de la fête sportive s'attache au maintien d'une authenticité et une véritable "fête de la Nation" peut se réaliser quand après le match de rugby "l'air austère des femmes de Bagnères rencontre l'excentricité des niçoises et que les deux camps se retrouvent autour de buvettes improvisées avec un mélange de savoureux accents et de coutumes" (1990 a : 77). Alors la "vérité" du sport, celle du rugby en particulier, s'établit quand existe cette "fête de famille" qui exige des sacrifices de présence pour être goûtée et une distanciation de sa popularité produite par la télévision, car il se transforme en "garden party" indécente en étant accaparé par ceux à qui il n'appartient pas, "Ces jours-là, disons-le franchement, le rugby est en fête et en même temps en berne" (ibidem : 84). La relation qui s'instaure là est abstraite, fictive, irréelle et le spectateur n'y est pas en réalité. La fête est désacralisée par la coupure instituée et par l'absence de mémoire collective qui concernent aussi les nouvelles pratiques comme la planche à voile ou le jogging qui segmentent le temps en 
instruisant une coupure entre le travail et le loisir, contrairement au rugby où "la rencontre n'est pas un instant isolé du reste de la durée, mais elle n'est parfois qu'un prétexte à une fête qui s'éternise" (ibidem: 148). C'est là pour Pierre Sansot, une manière de se distancier des théories de la sociologie du loisir de Joffre Dumazedier (1974, 1988), qui établit une séparation entre le temps contraint et le temps libre, négligeant le charnel, le savoureux, l'imprévisible, la multiplicité (1991 a: 19) car l'émotion du rugby par exemple ne dure pas seulement de quinze heures à dix-sept heures, "comme d'autres bâclent un amour de dix-sept à dix-huit heures" (1986 a : 94) pour passer après à une autre occupation.

La fête sportive, dans sa dimension sacrée, ne peut s'abstraire d'une durée incorporée, distanciée d'un vécu fragmenté du temps, et dans sa forme anthropologique, si elle se vit dans l'ici et le maintenant - dans l'instant du match - elle ne se réduit pas à ce moment. Sa signification ne vaut que par la mémoire qui donne du sens à l'attente de l'événement en développant l'imaginaire, par les affects provoqués par le match luimême, par leur prolongement lors de l'après-match. En aucun cas, la fête sportive se perçoit comme transgression d'un rite, et sa réalité tient à sa force conjonctive, à son ancrage dans une mémoire, dans une culture, ce qui en fait son authenticité, mais une authenticité très fragile car elle s'expose aux dérives modernistes.

L'entrée dans la légende

Sans doute eut-il été plus judicieux, pour respecter la pensée de Pierre Sansot, d'intégrer "l'héroïsation de certains êtres" dans la catégorie du sacré. Cependant, l'importance accordée au légendaire depuis quelques années mérite un développement séparé. Il s'agit là d'un point de vue qui excède un sport particulier et qui se rapporte en effet au champion ou au sportif de haut niveau sans spécification du sport pratiqué, c'est-à-dire à des êtres "héroïsés" appréhendés dans une conceptualisation échappant totalement aux thèses dominantes de la sociologie du sport, qu'il s'agisse de l'individualisme démocratique ou de la sociologie critique préoccupée par le rôle des idéologies politiques. La légende est préférée ici car elle nourrit l'imaginaire collectif et l'existence des sports à condition qu'elle jaillisse une nouvelle fois d'une authenticité alors susceptible de générer la rêverie. Le rôle instituant de l'imaginaire collectif s'affirme là, irréductible aux idéologies auxquelles il précède (1993 b : 414).

D'où la légende peut-elle naître par le sport, si ce n'est de cette fascination, de cet étonnement et de cette admiration des gens modestes agrégés autour de l'événement où les hauts faits sportifs peuvent être réalisés par des êtres hors du commun que vont répandre ensuite les journalistes ou les poètes (1993 c), ces êtres qui se transformeront alors en "êtres de légende" ? Un pays a besoin de " se ressourcer dans ses légendes" (1988: 272), et le sport lui en fournit les possibilités. Ces légendes peuvent être "constitutives d'une forme de socialité après coup" et s'engendrent par un processus méconnaissable dégagé de toute tentation structuraliste car "Il existe une mémoire obscure, tenace qui prend naissance (nous ne savons pas exactement où), qui épouse des modifications étranges et advient en nous " (1991 b : 74). Ces transformations du commun en légendaire tiennent à ce que ces sportifs ont su porter l'exception dans leurs disciplines à tel point qu'ils sont capables actuellement de supplanter la particularisation des cultures de classes, de créer un imaginaire collectif et d'inventer "une figure essentielle qu'elle se donne comme modèle, ou du moins, par rapport à laquelle elle prend ses mesures. Dans l'Antiquité, le Sage ; à l'aube de la chrétienté, le Saint; au début de l'industrialisation, le Prolétariat et l'Entrepreneur. Il nous semble 
que le sportif de haut niveau a pris aujourd'hui la relève de semblables figures imminentes" (1992 a : 188). Quelles sont les raisons qui font que ces êtres traversent le temps et qu'ils ne sombrent pas dans l'oubli?

Pour répondre à cette question, l'auteur majore deux dimensions : la possibilité de la mort réelle, de telle sorte que le commun peut par le sport se transformer en être d'exception, et la puissance domestiquée, au sens où l'apparente force que les sportifs dégagent, contraste avec des personnalités capables d'abnégation et de simplicité. La légende tient donc à cette fascination de l'exploit par l'imagination que les sportifs suscitent, née de la jonction dans les mêmes êtres de la permanence de traits ordinaires et de la réalisation d'actions extraordinaires. Cette combinatoire commun-exception prend la forme d'une combinaison sacré-non-sacré, "qui ne consisterait pas en l'intrusion d'une transcendance mais dans la sédimentation d'une mémoire collective, dans la capacité des hommes à fabuler, à dire et à produire l'extraordinaire" (1990 b : $60)$.

L'admiration du peuple pour l'exploit et les pratiques ordinaires que Pierre Sansot veut réhabiliter, tient à la proximité de la mort réelle de l'alpiniste, du coureur automobile, du coureur du Tour de France ou du joueur de rugby, ou encore à la symbolique en athlétisme d'être le plus haut du monde. La présence des gens modestes est constamment soulignée, qui, rassemblés en grand nombre, font "trembler" la cité, la montagne ou l'histoire, font naître les légendes "comme celle du Tour de France ou de certaines coupes de football, de certaines grèves dures" (1991 a: 16), donnent des surnoms toujours vivants à ces êtres victorieux ou non d'épopées qu'ils aiment réciter, ces demi-dieux dont la souffrance les rapprochent de la mort comme le rappellent des expressions "descendre à tombeau ouvert", ces rejetons d'une race de Titans qui s'appelaient "l'Aigle de Tolède" (F. Bahamontès), ou "Le Fou Pédalant" (F. Kubler), ou "Le Blaireau" (B. Hinault) (ibidem, 201), ou au rugby, ces héros des "combats homériques" en danger de mort réelle et non pas symbolique dès qu'ils sont en possession du ballon dans ce sport qui ne crée pas de stars mais dont les figures risquent bien de disparaître face aux transformations qu'il subit (1988: 272 ). Légende encore des joueurs de football évoquée par les journalistes et qui peut naître soit du contraste entre la réussite sportive des joueurs et leurs origines modestes (comme Trésor venu de Corse avec 300 francs et qui fit son apprentissage aux Minguettes), soit de la fascination par la grâce des "dons" qui les situent "au-dessus des mortels" (1991 a : 145), ou encore de la modestie des joueurs de rugby par exemple, rompant avec l'image des "piliers monstrueux", expression loin d'être méprisante pour Pierre Sansot qui en les rencontrant a pu noter comment "la réalité ne démentait pas la légende : simplicité, modestie, gentillesse les caractérisaient. Celui qui les avait conquis en amitié pouvait compter sur leur parole" (1990 a : 99). Assurément sa sympathie est immense pour "ces vieux grognards qui ont risqué leur intégrité physique, peut-être leur vie (...). Il y a aussi ce qu'ils sont devenus et qui atteste qu'ils furent les témoins de batailles grandioses. Des drôles de gueules, de nez brisés, des oreilles en chou-fleur, une masse de chair qui a pris monstrueusement de l'embonpoint, une jambe que l'on traîne ... " (1990 a : 171-172).

Ces héros hors du commun sont la source de nos rêveries et s'intègrent à notre imaginaire collectif parce qu'ils font partie d'une la mémoire que Pierre Sansot entend signifier et faire resurgir, non pas par goût des survivances, ce serait se méprendre sur ses objectifs, mais seulement comme une mise en scène d'un monde qui est en train de 
disparaître, sans qu'il y ait là un quelconque militantisme passéiste (1991 a : 21), car les légendes tendent à disparaître dans la modernité.

L'entrée dans la modernité

61 Cette vue poétique du sport fondée sur une théorisation du sacré et du légendaire ancrée à une authenticité des rites fait-elle de Pierre Sansot un contempteur de la modernité ? Comment saisit-il à propos des sports cette articulation très féconde en sciences sociales entre la tradition et la modernité ? Comment perçoit-il les transformations des sports faits d'une mémoire, comme le rugby? Quelle place accorde-t-il à ce sport dans le champ des sports sans recourir à une quelconque approche structurale? Son irrespect envers "les sports narcissiques" caractérisés par le plaisir est-il irréductible puisque, le rugby se fonde aussi sur cette sensation?

Esquisser une réponse à ces questions, c'est prendre le rugby comme un analyseur des autres sports, car il semble bien que ce soit à partir de lui que se construit son regard sur les autres sports, notamment sur leur place dans la modernité, car si le rugby subit son emprise, il s'érige cependant en possible contre-modernité.

Le rugby apparaît en effet comme un sport en tout point opposé à la modernité, par son "anachronisme", par sa forme de "résistance au temps", par la perpétuation d'une forme d'amateurisme contre le professionnalisme, par la possible rupture qu'il établit "avec le statut de spectateur exilé des idoles" (1990 a : 11). Son statut particulier tient à la tradition, parce qu'il a su conserver des valeurs comme l'honneur, le courage, l'exaltation de la vengeance, ou la réponse immédiate à l'offense (ibidem: 56), à la rudesse du jeu, à "un goût de la simplicité, un respect de la vérité qui met un frein à la rage de paraître" (ibidem : 17), vérité qui ne peut être théâtralisée, car elle se situe loin des faire-semblants et des tricheries grâce aux apprentissages incorporés très tôt par des entraîneurs veillant à transmettre des règles morales, peu communes aux autres sports. Contre-modernité encore, car il est en rupture avec l'idéal olympique inventé et propagé par l'aristocratie, et sous ses habits empruntés à la modernité "réapparaît au rugby, plus qu'ailleurs, une foule de braconniers, des personnages dignes du Roman de Renart, tout un peuple pour lequel la ruse (arme des faibles) a toujours été une vertu" (ibidem : 105). Le poids de la tradition et des valeurs conservées opposées aux valeurs actuelles paraissent préserver le rugby d'une dérive dans la modernité, mais cette dernière l'imprègne et les contours traditionnels purs s'estompent et ce sport se transforme lui aussi.

La méthode utilisée ici pour saisir cette dialectique tradition-modernité est rigoureuse et n'établit pas de frontières nettes entre ces deux moments d'une évolution qui serait facilement discernable. Elle montre leur conjonction, leur interpénétration et la transformation dans leur essence, de "l'esprit" des joueurs et de celui du spectacle. Inévitablement la tradition est bousculée, brouillée par l'évolution exacerbée du sport vers la performance, et l'enfance, lieu d'imprégnation des apprentissages, subit ces métamorphoses car les jeunes "se laissent happer par la modernité, à travers la musique et la B.D. (...). Les meilleurs veulent devenir plus performants. Une pareille modification de statut, des aspirations de l'enfant me semble aller de pair avec l'évolution du rugby (...). Et il n'est pas étonnant que le rugby, à son tour, se soumette aux principes de raison, de réussite, d'efficacité" (ibidem : 14).

Ces changements peuvent transformer l'identité même de ce sport, à tel point que Pierre Sansot se demande si les joueurs sont encore "vraiment rugby" car l'autre génération "témoignait du respect, ils se rencontraient de souverain à souverain, selon 
un certain protocole", et être rugby c'était renouveler sa licence jusqu'à 55 ans, n'accepter "l'extrême onction que si le prêtre arrive à leur chevet, une hostie à la main et un ballon ovale dans l'autre (ibidem : 32-33). Les entraîneurs, ces "peloteurs d'âmes", ces "tripoteurs de conscience", ont donc une responsabilité, et certains d'entre eux, en devenant professeurs d'Éducation Physique risquent de "cérébraliser" ce qui est "instinct, désir" (ibidem: 116), ou de développer l'idée de plaisir et non plus celle d'abnégation d'autrefois. Même les rapports aux corps se modifient, l'entraînement se rationalise, les corps deviennent plus harmonieux et "les cicatrices du temps sont rarement visibles" (ibidem : 172), les usages du corps deviennent plus subtils dans le jeu (ibidem : 151).

Les nouveaux pratiquants lui paraissent avoir moins le sens collectif, sans cependant qu'ils soient repliés sur eux-mêmes ou sur leur famille. Ils s'intègrent en réseaux et sont soucieux de diététique, ce qui met fin au mythe du steack saignant (ibidem: 156-157). Les différences entre les sports tendent alors à s'estomper et si les nouvelles pratiques et le rugby ne s'opposent plus puisque l'hédonisme est présent, des différences demeurent cependant sur le plan de valeurs : "Les premiers disent plaisir, liberté, les autres vantent les vertus du travail et du mérite" (ibidem: 150). La modernité étend donc ses valeurs sans toutefois anéantir le substrat, le noyau dur, qui fait la "culture" de certains sports, irréductible à un bouleversement total. Tradition et modernité se conjuguent dans l'instant pour engendrer plutôt des mutations qu'une réelle révolution.

Mais Pierre Sansot analyse les ruptures que le rugby peut connaître sous l'effet de la modernité, liées aux formes de management des sports telles que la médiatisation, le professionnalisme ou le sponsoring, pouvant l'entraîner vers des formes sportives communes aux autres sports (ibidem: 157 sq.). Déjà la médiatisation du Tournoi des Cinq Nations tend à dévaloriser les prestations moyennes et le sponsoring trouverait dans le rugby des valeurs auxquelles s'attache l'industrie moderne, "la tonicité, l'entraînement à la douleur, la rage de vaincre, la solidarité", mais transformé en "Intermarché" ou "La vie claire", le rugby perdrait son âme (ibidem : 162). De même, le professionnalisme comporte des dangers de rupture par rapport aux conceptions fondamentales reposant sur "l'engagement, la ferveur, la gratuité, une manière de vivre au-dessus, en dehors du fonctionnel" (ibidem: 161), sans compter les conséquences sur les joueurs de rugby "brûlant les limites du surhumain, des êtres suréquipés au plan physiologique et mental" risquant une mort provoquée par la "surpuissance de ses adeptes" (ibidem: 162). Internationalisation, modernité ont des conséquences sur la tradition dont Pierre Sansot souhaite le maintien et il est satisfait de la résistance qu'offre le rugby à ces tentations. Ce sport apparait bien isolé de l'ensemble des sports et s'oppose à une rationalisation à outrance défigurant son essence. En cela, l'oeuvre de Pierre Sansot fourmille de rappels constants à l'histoire, nous invitant à nous interroger sur ce que nous devenons et sur ce que nous faisons, non pas dans un désir de retour passéiste ou nostalgique à d'anciennes manières de faire, mais, à la manière de Georges Balandier (1985), par un "détour" qui montre par comparaison, l'effacement dans la contemporanéité de structures et leur renouvellement sans que la mémoire soit absente de ces turbulences, en nous montrant alors la mythologie du neuf comme il le fait en comparant le rugby à d'autres sports. L'actualité confirme bien toutes ces interrogations, puisque des valeurs comme l'amateurisme ou l'attachement à une culture locale paraissent bien compromises au regard de projets alliant internationalisation, sponsoring et professionnalisation. Deux 
projets en effet sont suffisamment avancés pour qu'ils parviennent à terme à modifier la tradition. Le premier vise à créer une superligue franco-britannique constituée d'une dizaine d'équipes professionnelles regroupant les meilleurs joueurs de chaque pays et s'appuie sur la médiatisation (la chaîne satellite BskyB) et la participation financière des grands brasseurs de bière, le second s'inspire des shows américains avec divertissements musicaux inclus et est piloté par l'ex-entraîneur de l'équipe de France, Jacques Fouroux qui le présentera en novembre à la presse internationale (L'Express, 1994).

La distanciation établie par Pierre Sansot vis-à-vis des nouvelles pratiques se justifie par le dédoublement opéré entre le pratiquant centré sur ses sensations et un environnement jamais considéré dans sa sacralité, dans son caractère mythique qui est utilisé seulement pour satisfaire ses fins. Dans ce contexte, la montagne, la rue, les stades, les paysages pourtant chargés de symboles n'ont plus de signification en soi. Ils sont effacés de leur pouvoir d'établir un rapport soi-monde et ne valent plus qu'en fonction du plaisir narcissique qu'ils procurent. Alors sa sensibilité le porte davantage vers des sports dont le sens s'ancre à une culture populaire, dont la signification se lie à une mémoire créatrice d'un imaginaire irréductible à la seule fonctionnalité rationalisante et individualisante. Et puis les pratiques traditionnelles véhiculent elles aussi des valeurs désignées facilement de "nouvelles", comme le goût de l'aventure, et nul n'est besoin de conquérir de nouveaux espaces pour assouvir le "goût de la vastitude" qui peut "s'accommoder d'un amour des limites", et le goût de l'illimité est ressenti par exemple en rugby par ceux qui perforent ou contournent une défense adverse" (1990 a : 153). L'accélération de l'histoire privilégie le nouveau et favorise la mythologie du neuf, la surestimation du moderne et nous rend inattentifs à des permanences, voire à des résurgences de l'archaïque (ibidem: 143). Bref, nous le sentons, l'intérêt se porte vers les sports pour lesquels la culture populaire s'exprime de façon charnelle et Pierre Sansot tient à distinguer le football du rugby. Si le premier est un sport populaire, le rugby ne l'est pas si on entend par là "un sport universellement répandu dont les adeptes sont fort nombreux (...). Populaire, cela peut vouloir dire qui envahit les rues, qui met en liesse, pendant des journées entières, toute une population, qui force les barrages de la censure et des bonnes moeurs. C'est ce qui se passe pour le football au Brésil. D'autres pratiques sportives (mais jamais le rugby) suscitent cette atmosphère de carnaval. L'avant match, l'après match d'un partie de rugby ont de la peine à se prolonger" (ibidem: 49-50). L'assimilation des sports au qualificatif de "collectif" comme ces deux sports, ne conduit pas à leur homogénéisation, car si l'essai aboutit parfois à un essai collectif, Pierre Sansot imagine mal "un but collectif", et les réactions aux essais marqués sont bien différentes du football, témoignant bien de l'absence d'individualisme car ici personne n'endosse le rôle "du héros ou du surhomme" (ibidem : 41).

$\mathrm{Au}$ terme de cette présentation de la vision poétique du sport de Pierre Sansot, nous avons bien conscience d'avoir opéré un découpage méthodologique en trois catégories, le sacré, la légende et la modernité éloigné d'une fidélité à l'oeuvre originelle qui mêle ces thèmes. Finalement les différenciations établies entre les sports ne puisent dans aucune référence structurale, mais s'appuient sur leurs capacités à provoquer la rêverie toujours liée au concret et à en montrer ses possibilités structurantes. La théorisation, jamais présentée comme telle neutralise tout ce qui fige un imaginaire et une symbolique dont les sources s'accrochent au contraire à l'événement présent et non pas à une quelconque mythologie puisée dans un fond archétypal. Cela n'interdit 
nullement le retour à l'histoire ou à la mémoire qui deviennent des recours moins pour appeler à la nécessité pour la modernité de retrouver ces signes que pour montrer les résistances qui lui sont opposées, même si son emprise risque de conduire le sport le plus traditionnel, comme le rugby, à une uniformisation avec les autres sports à cause du management moderne et de l'appel au profit généralisé dont il devient l'objet. Le sensible, dans ce sens risque bien de devenir une valeur productive, utilitariste, source de rentabilité pour la commercialité en tout point opposée aux valeurs que lui attribue Pierre Sansot. En effet, un "usage cynique" de ses analyses peut se produire, comme cela a été le cas pour d'autres recherches en sociologie, comme celles de Pierre Bourdieu par exemple qui entend par là des usages "qui consistent à chercher dans l'analyse des mécanismes sociaux des instruments pour "réussir" dans le monde social (c'est ce qu'ont fait certains lecteurs de La Distinction, en traitant ce manuel comme un manuel de savoir-vivre) ou pour orienter ses stratégies dans le monde intellectuel", usages se différenciant d'un "usage clinique qui offre un moyen de connaître et de comprendre les autres (soi-même)" (Bourdieu P., Wacquant L. J.D., 1992 : 182-183). De même la fascination de Pierre Sansot pour le sport, loin de se circonscrire à une perspective phénoménologique favorisant le rapport soi-monde, pourrait alors être exploitée à des fins économiques, car l'émotion, le rêve, l'imaginaire, deviennent des vecteurs de rentabilité, tout comme les loisirs machandisés cultivent le goût du sensible qui devient une "valeur" à satisfaire produisant du plaisir s'incluant alors dans une logique de consommation pervertissant ce qui pour lui fait la richesse du sport.

Les rêveries poétiques de Pierre Sansot sont bien moins anodines que ne le laisse supposer un premier regard. Sa proximité intellectuelle de Gaston Bachelard tend à nous faire comprendre toute la portée critique de la poésie appliquée au sport, ceci dans un sens identique à celui que Georges Canguilhem accordait à Gaston Bachelard qui voyait dans la source des rêves, des images, des illusions, une puissance de négation à la raison, raison nécessaire pour appréhender la réalité du monde toujours remise en question, "C'est la permanence de ce pouvoir originaire, littéralement poétique, qui contraint la raison à son effort permanent de dénégation, de critique, de réduction. La dialectique rationnelle, l'ingratitude essentielle de la raison pour ses réussites successives ne font donc que désigner la présence dans la conscience d'une force jamais lassée de divertissement du réel, d'une force qui accompagne toujours la pensée scientifique non pas comme une ombre, mais comme une contre-lumière" (Canguilhem G., 1957). En ce sens, les rêveries sportives de Pierre Sansot le conduisent bien à une critique du sport et à celle des formes prises par les transformations que subissent ceux qui s'attachaient à une tradition, à tel point qu'il voue une passion aux pratiques qui se rapprochent du jeu, "Je me demande comme bien d'autres, si nous ne confondons pas à tort le sport et le jeu. Le premier exige tellement de sérieux, se fonde sur un travail forcené qui confine à l'ennui. J'associe le second à une entière liberté, à une ivresse proche de l'enfance et du poète; mais nous n'aurions pas le courage d'avancer que la sphère du sport a pris la place de celle du jeu (en voie de disparition) ..." (1990 a : 22).

Variations herméneutiques

71 Il faut s'accorder avec Pierre Ansart quand, en conclusion du chapitre intitulé "Ordre et désordre" de son ouvrage Les sociologies contemporaines (1990:267), il repère dans les approches dynamiques, outre la sociologie de Georges Balandier et celle d'Alain Touraine, celle d'auteurs qui sans constituer véritablement une école, sont ouverts à l'anthropologie, à l'histoire, aux "complexités et aux multitudes des changements" et 
classe dans un courant sensible "à la culture et aux imaginaires", Pierre Sansot auprès d'autres auteurs comme A. Akoun, J. Duvignaud, P.-H.Chombart de Lauwe, J. Dumazedier, G. Durand, G. Namet, P.-H. Jeudy, J.-P. Sironneau et lui-même. Pierre Ansart précise également la place qu'occupe Pierre Sansot dans le champ des recherches sur les idéologies, les imaginaires sociaux et les représentations, champ en développement comme l'est également celui des communications, de la vie quotidienne, de l'immigration ou de la sociolinguistique, et montre comment il se situe, avec les études critiques des représentations de groupe de Jean Baudrillard ou de Denise Jodelet, dans une approche des représentations sociales surmontant les frontières entre l'approche sociologique et l'approche en terme de psychologie sociale (ibidem: 317-319). Mais est-il possible, tout en partageant cette catégorisation, de spécifier la place de Pierre Sansot dans le champ de la culture et des imaginaires sociaux? En effet l'appartenance à un champ, repérable par des concepts communs permettant de l'identifier, ne signifie pas un recours à la même épistémologie, comme en témoigne la distance prise par Pierre Sansot vis-à-vis de certaines conceptions de Gilbert Durand ou de Joffre Dumazedier par exemple. La référence au même thème, l'imaginaire social, produit des travaux différents et conduit à des interprétations variées, parce que les bases théoriques de la connaissance du social s'appuient sur des épistémologies contrastées. Il paraît alors nécessaire de montrer le positionnement singulier de Pierre Sansot dans ce champ qui a été identifié comme celui d'une anthropologie poétique attentive au sensible et à l'imaginaire, et d'apprécier sa singularité, et aussi sa proximité, par rapport aux auteurs auxquels il se réfère, comme Gilbert Durand, ou que l'on pressent comme Michel Maffesoli, ou encore sa distance vis-à-vis de la sociologie politique, qui elle aussi fait appel à la notion d'imaginaire social. Enfin, il faut aussi voir de plus près comment son éloignement proclamé de la sociologie de Bourdieu, peut aussi conduire paradoxalement à quelques rapprochements.

Ici encore, il existe un risque lié à la volonté de comparer des auteurs dont l'importance est attestée dans un champ scientifique, qui consiste à réduire la part d'innovation de l'auteur dont on cherche à comprendre l'originalité des positions théoriques en esquissant la mise à jour des fondements de sa pensée, obligatoirement réducteurs, simplificateurs et arbitraires, puisqu'ils tiennent compte davantage de l'état de culture de celui qui tente cette interprétation, que de la réalité même de la pensée de l'auteur interrogé et des auteurs du champ dont l'accessibilité comporte inévitablement une grande part de mystère. Il s'agira de déconstruire ici les irréductibilités des théories de la connaissance du social, persuadé, comme Jean-Michel Berthelot l'a démontré (1990), qu'il faut dépassionner les conflits - ce que souhaite la communauté - en montrant que chaque auteur peut conserver une singularité dans sa théorie du social, comprise comme un ensemble faisant unité entre la construction théorique, la preuve et l'interprétation, tout en trouvant des transversalités dans la variabilité des schèmes d'intelligibilité, et ceci malgré les hétérogénéités que tendent à maximiser les différentes écoles générant exclusions irrévocables et polémiques indépassables, schèmes qui peuvent d'autre part se combiner chez un même auteur. Il ne s'agit pas de relativiser les théories de la connaissance, mais de montrer, en s'appuyant sur la démarche de Jean-Michel Berthelot, que les auteurs tendent, pour affirmer leurs thèses, à privilégier certains axes et à en neutraliser d'autres. Ainsi l'anthropo-sociologie de Pierre Sansot, va-t-elle servir ici d'analyseur d'un mode d'approche du social fondé sur un schème herméneutique dont il s'agira de montrer les différences avec des approches 
fondées sur l'imaginaire, tantôt saisies dans celles qui paraissent proches (les analyses sur l'imaginaire de Gilbert Durand), ou plus éloignées (les analyses politiques de type marxisantes reposant sur les idéologies),ainsi que les ressemblances avec des théories qu'il neutralise, celles de la culture réalisées par Pierre Bourdieu par exemple, en entrant donc dans le conflit des herméneutiques (Ricoeur P., 1975).

Le cercle herméneutiqueLe conflit des interprétations

Paul Ricoeur dans son ouvrage De l'interprétation (1975), vise par une démarche philosophique, à analyser "la consistance du discours freudien", entreprise qui soulève trois types de problèmes: épistémologique d'abord (qu'est-ce qu'interpréter?), de philosophie réflexive ensuite (quelle compréhension nouvelle de soi révèle cette interprétation?), dialectique enfin (l'interprétation freudienne de la culture est-elle exclusive de toute autre ?). Concernant le premier problème qui est aussi au fondement de la pensée phénoménologique de Pierre Sansot, l'auteur entend discuter les relations entre le symbole et l'interprétation, en montrant le double sens du symbole, comme région du langage donnant lieu à des significations complexes et comme sens qui se cache dans un sens immédiat, double sens qui n'appartient pas exclusivement à la psychanalyse, mais aussi à la phénoménologie (notamment de la religion) qui s'intéresse aux grands symboles cosmique de la terre, des eaux, aux mythes et qui sont "une manière pour l'homme de se mettre en rapport avec la réalité" (1975: 16). Cependant, la phénoménologie se différencie de la psychanalyse car elle ne connaît pas le symbole comme distorsion de langage mais le considère comme affleurement du sensible dans l'imagination, le geste, le sentiment. Le problème d'un conflit des herméneutiques se pose donc pour Paul Ricoeur, herméneutique qu'il définit comme "la théorie des règles qui président à une exégèse, c'est-à-dire à l'interprétation d'un texte singulier ou à un ensemble de signes susceptibles d'être considéré comme un texte..." (ibidem : 18). L'herméneutique est tantôt envisagée comme "la manifestation et la restauration d'un sens qui m'est adressé à la façon d'un message, d'une proclamation ou, comme on dit parfois, d'un kérigme", tantôt pensée comme "démystification, comme une réduction d'illusion. C'est de ce côté que se range la psychanalyse, du moins en première lecture" (ibidem : 35 ). Cette oscillation entre la démystication et la restauration ou la "récollection" de sens, s'affirme aussi dans l'approche de Pierre Sansot qui défend bien le projet d'une herméneutique phénoménologique opposé à celui du soupçon. Ce projet consistant donc plus à décrire, en révélant l'objet implicite dans le rituel, qu'à le réduire, réduction impliquant la recherche d'explications de type causaliste ou historique. Cette "description" s'opère au moyen d'une attitude neutre réglée par l'épochée, par cette suspension de la réalité absolue et qui fait abstraction des savoirs constitués par les sciences sur les phénomènes, par cette mise hors jeu de notre engagement dans le monde objectif, rapport immédiat qui précède toute réflexion. L'intention phénoménologique, comme la pratique Pierre Sansot, est bien d'abord descriptive, recherche d'une forme sensible qui présuppose l'existence du phénomène (réduction éidétique). Ce projet s'oppose à celui de l'interprétation comme exercice du soupçon qui vise à réduire des illusions et les mensonges de la conscience et que Paul Ricoeur reconnaît en Marx, Nietzsche ou Freud, qui ont en commun de mettre en avant la conscience comme "fausse conscience". L'objectif ne revient plus à chercher la conscience du sens, mis à "en déchiffrer les expressions (...) tous les trois croient (...) une science, médiate du sens, irréductible à la conscience immédiate du sens" (ibidem : 41) et convergent vers la procédure de la mystification en visant la réduction de l'illusion. Or, toutes les recherches de Gilbert Durand s'articulent semble-t-il, sur cette 
volonté, commune à Pierre Sansot, de donner toute sa valeur à une herméneutique comme "récollection de sens", et de contribuer à une "remythisation" (1984 : 109). Cela les unifie autour du "cercle herméneutique" et les différencient de ceux qui en sortent, comme la conception marxisante de la représentation, puisque le schème d'intelligibilité dominant est là de type dialectique, neutralisant le pouvoir explicatif du schème herméneutique au sens où, comme l'écrit J-M. Berthelot, "le fondement du sens, sa base matérielle, dans la structure sociale et la pratique historique constitue une rupture originale avec le schème herméneutique (...). En instituant les représentations comme reflets des conditions de vie matérielle des hommes, Marx brise le cercle de façon originale. Il ne tombe pas dans le réductionnisme du matérialisme classique : les représentations ne renvoient pas aux choses, dont elles ne seraient que des émanations ou des traces sensorielles, mais résultent d'une élaboration trouvant dans la praxis sociale son fondement" (1990: 98-98).

La puissance de "l'imaginatrice"

74 Gilbert Durand opère cette distinction à propos de Freud en refusant de réduire le symbole à un épiphénomène, en considérant comme réductive une herméneutique de type archéologique qui plonge dans le passé biographique ou sociologique, et plaide plutôt pour une herméneutique eschatologique amplifiant le symbole, "appel à l'ordre essentiel", "réminiscence", (1984: 109). Ici se retrouve un fondement de la pensée de Pierre Sansot, puisque cette amplification s'opère chez ces deux auteurs par une valorisation de l'imaginaire comme puissance organisatrice de l'entendement et qu'ils se réfèrent pour cela aux thèses bachelardiennes afin de contester l'objectivisme et le rationalisme, et sans doute Pierre Sansot s'accorderait-il avec cette réflexion de Gilbert Durand "Le plus humble des mots, la plus étroite compréhension du plus étroit des signes, est message malgré lui d'une expression qui nimbe toujours le sens propre objectif" (1992: 496). En outre Gilbert Durand, pour construire son "Nouvel Esprit Anthropologique" (1979), se réclame du projet de Gaston Bachelard quand il a entrepris de réaliser le "Nouvel Esprit Scientifique", mais il entend le faire en évitant "l'enthousiasme scientiste de la gigantesque mutation du Nouvel Esprit Scientifique" (ibidem : 55) pour se centrer sur le dévoilement de l'essence et non sur la dénonciation du masque ou sur la défense du principe de causalité. Il souhaite donc majorer, comme l'a montré l'anthropologie scientifique, la permanence et la validité de la "pensée sauvage", "plus universalisée dans l'espèce humaine que le perpétuel bouleversement des théories et des méthodes scientifiques" (ibidem: 55). D'où l'importance attribuée par Gilbert Durand au mythe, au numineux, aux archétypes, aux conversions de l'anthropologie telles que Edgar Morin les a proposées (ibidem : 221), au pluralisme, à la recherche de "l'Adam primordial" (ibidem : 232), à une science de l'homme ouverte sur la Tradition (valeurs, coutumes, rites, mythes, les "leçons" des légendes et des histoires) (ibidem : 223). Et Gilbert Durand conseille aux étudiants, ce que Bachelard recommandait à ses propres étudiants, c'est-à-dire la lecture des poètes, "ces mainteneurs du mythe", qui "permettent de lever le couvercle étouffant de notre ethnocentrisme technocratique, bureaucratique et scientiste" (ibidem : 235).

Gilbert Durand aime à se décrire comme "Bachelard de gauche", tendance qu'il est possible aussi semble-t-il, d'attribuer à Pierre Sansot, au sens où comme lui, il accentue moins le "réformisme rationnel du maitre" que la rupture révolutionnaire de "l'axe de la pensée poétique par rapport à la science" (Durand G., ibidem : note 109: 185). C'est bien de l'impossibilité de "dissocier le schéma des liaisons axiomatiques et le contenu intuitif de la pensée" propre à Gaston Bachelard, dont s'imprègnent Pierre Sansot et 
Gilbert Durand. Ce point de vue s'accentue cependant chez Pierre Sansot dont la poétique onirique maximise l'association que faisait Bachelard entre la rêverie et la matière (Bachelard G. 1942 : 4), rêve qui précède la contemplation, car "avant d'être un spectacle conscient, tout paysage est un espace onirique" (ibidem:6), images qui procurent un bien être corporel par les traces que laissent les rêves, imagination dont la faculté n'est pas de former mais de déformer les images fournies par la perception, elle est surtout la faculté de nous libérer des images premières, de changer les images", c'est le changement qui produit l'imagination, sans lui il n'y a pas "d'action imaginante" (...). Grâce à l'imaginaire, l'imagination est essentiellement ouverte, évasive" (Bachelard G., 1943 : 7). C'est bien là que se trouve aussi un trait important de la pensée de Pierre Sansot: l'imagination comme invitation au voyage, comme élancement, comme dynamique, correspondant à ce qu'écrit encore Gaston Bachelard, "Enfin le voyage dans les mondes lointains de l'imaginaire ne conduit bien un psychisme dynamique que s'il prend l'allure d'un voyage au départ de l'infini. Dans le règne de l'imagination, à toute immanence s'adjoint une transcendance. C'est la loi même de l'expression poétique de dépasser la pensée " (ibidem : 12).

L'imagination créatrice est commune aussi à l'anthropologie de Gilbert Durand, quand il développe, à partir des travaux de Henry Corbin (1976), la notion d'imaginal chère également à la sociologie de Michel Maffesoli. Cette notion "d'imaginatrice" développée par Henry Corbin, c'est-à-dire des visions imaginales comprises comme un ensemble d'images sensibles, nullement visionnaires et qui font connaître un objet, représente au sens où l'entend l'auteur en s'appuyant sur la phénoménologie, une imagination réellement "active", un monde intermédiaire s'intercalant entre deux autres modes de perception auxquels la connaissance du monde ne peut se réduire, la perception intellectuelle et l'univers des sens, c'est-à-dire le monde des "Idées-images, des Figuresarchétypes, des corps subtils, de la "matière immatérielle", un monde aussi réel et objectif que l'univers sensible et l'univers intelligible "où le spirituel prend corps et où le corps devient spirituel" (Corbin H., ibidem : 11-13). Certainement faudrait-il associer au développement de cette pensée, la sociologie de Michel Maffesoli, dans laquelle le sensible occupe une dimension majeure revendiquée à cause de son exclusion par la Raison et par la modernité, et qui accorde alors une attention particulière aux thèmes qu'elles excluent, comme le rôle des émotions, des affects, des sensations, de l'imaginal comme possible réenchantement du monde contre la technique (1990: 108 sq.), ensemble de thèmes dont la présence est également très forte dans l'anthropologie de Pierre Sansot. Reconquérir le sensible, pour Pierre Sansot comme pour Michel Maffesoli, c'est avoir accès au plaisir, à la socialité, à l'être ensemble, à l'insignifiance, dimensions essentielles dans la vie quotidienne. Bien sûr des variantes existent entre ces deux types d'approches. Les références à la modernité n'atteignent pas dans les recherches de Pierre Sansot le degré critique que leur accordent celles de Michel Maffesoli qui oppose la rationalité, l'instrumentation, l'aliénation, à l'empathie, à la culture du présent, à la liberté interstitielle et au vitalisme. Mais ici encore, l'imaginaire et l'approche compréhensive sont valorisés contre les vues causalistes, positivistes et scientistes.

Variations autour du schème herméneutique

Il a été question jusqu'à présent, à partir des travaux de Pierre Sansot, de montrer des constantes communes au schème herméneutique - notamment l'importance accordée à l'imaginaire - ceci à partir d'approches différentes quant à son opérationnalisation. La filiation théorique des auteurs à Gaston Bachelard, Mircéa Eliade ou Roger Caillois, et 
leur distance vis-à-vis d'une science de l'homme objectiviste, les intègre dans une phénoménologie poétique, sensible, pluraliste, favorisant l'idée de numinosité comme mode d'accès à la connaissance. Cependant, en poursuivant le projet de J.-M. Berthelot, peut-être faudrait-il s'efforcer de décrypter, au sein même de ce schème herméneutique qui les agrège, d'autres schèmes qui contribuent, tout en permettant cependant leur identification, à montrer des différenciations singularisant ces épistémologies dans ce champ. En effet, Pierre Sansot nous alerte sur des divergences éventuelles: "D'autres que nous, ont soutenu avec brio, que l'imaginaire ne s'est pas tari et l'on évoque le retour de Hermès ou de Dionysos. Telle n'est pas notre hypothèse. S'il nous fallait des garanties théoriques, nous préférerons croire avec L. Sfez que certains dispositifs symboliques se mettent à l'oeuvre dans telles ou telles configurations culturelles et socio-économiques. Nous disons encore autre chose qui rend inutile, nous semble-t-il, le recours à des archétypes ou l'existence d'une réserve de grands mythes, trésors inentamés de l'humanité. Une culture, une société, voire un micro-groupe ne peut pas se réfléchir, ne pas se représenter, sous forme d'images, de récits, de gestes lourds, ce qu'il vit ou ce qu'il pratique. Ces images sont plus ou moins claires, plus ou moins superbes. Un stade vibre et il s'émeut de sa propre rumeur et, $\hat{o}$, énigme, le béton se met à tanguer. La lourde enceinte ressemble à un bateau ivre (...). Non, les mythes (éternels ou archaïques) ne viennent plus hanter nos existences ou nos inconscients, même quand ils se recyclent sous la forme des images plus médiocres de la publicité" (1986 a : 207). Sans doute cette réflexion s'inscrit-elle dans une volonté de se soustraire en partie des théories de Gilbert Durand? (1979, conclusion intitulée" Le nouvel esprit anthropologique ou le retour d'Hermès : $236 \mathrm{sq.)}$.

Une première rupture paraît donc devoir être relevée. Elle concerne le recours aux mythes dont Pierre Sansot souhaite se détacher, même s'il s'en réfère dans sa constitution du légendaire. Il apparaît plus séduit par Bachelard que par Eliade, même s'il recourt aux thèses de ce dernier pour donner du sens au sacré (1991 a: 46). Peu importe pour lui les invariants, c'est le présent attaché à une culture ancrée dans le concret qui l'emporte pour donner sens aux actes dans la vie quotidienne neutralisant en cela la reconnaissance de la puissance de l'archétype, centrale dans l'oeuvre de Gilbert Durand qui le définit en s'appuyant sur la psychanalyse, surtout sur Jung, comme une "zone matricielle de l'idée", comme un "substantif symbolique" constituant "le point de jonction entre l'imaginaire et les processus rationnels" (1992 : 62-63 ; 1984). Comme tel l'archétype se différencie du symbole par son manque d'ambivalence, et Gilbert Durand prend l'exemple de la roue qui est "l'archétype du schème cyclique et on ne voit pas quelle autre forme imaginaire on pourrait lui donner, tandis que le serpent n'est que le symbole du cycle, symbole fort et polyvalent" (ibidem: 63). Le symbole par opposition à l'archétype est riche de sens différents. On comprend alors la préférence accordée par Pierre Sansot aux "dispositifs symboliques", probablement parce qu'ils ne sont pas substantialisés du fait de leur jonction avec un social en construction permanente, mais profondément dynamiques et ouverts, consubstantiellement liés aux contextes - au sens où l'entendait Lucien Sfez (1978). Alors, la référence à l'imaginaire ne lie plus des univers semblables dans les approches de Pierre Sansot et dans celles de Gilbert Durand. Ainsi, si le côté eschatologique est présent dans ces deux types de recherches, Pierre Sansot n'estime pas nécessaire la présence de l'archétype et reconnaît la nécessité de l'archéologie, relativisée par Gilbert Durand. Cette différenciation s'illustre par exemple dans les interprétations du sport ou des conduites du corps. Si les analyses du sport de Bernard Jeu par exemple 
peuvent a priori rappeler celles de Pierre Sansot par une sensibilité commune à l'émotion par exemple, elles s'en distinguent fortement par l'absence en ce dernier de tout désir classificatoire des sports fondé sur le mythe, et par l'absence de références à une société primitive dont les formes seraient capables d'expliquer certains phénomènes propres au sport moderne comme la violence (Jeu B., 1987, 1992). Les approches de Pierre Sansot en matière sportive convergent plutôt vers une clinique phénoménologique non causaliste, sans référence à l'archétype, comme la réalise Jacques Birouste qui, par la notion d'aesthésie, reconnaît la pratique sportive comme le lieu de gestation des impulsions singulier à chaque être, conjoignant le somatique et le spirituel, appartenant au mouvant, au dynamique, au transitoire, avec une reconnaissance de la numinosité dans la dimension glorieuse du sport fondée sur les héros et les champions ou sur la résonance entre le sportif et les éléments matériels proches qui constituent une "ambiance", par exemple le rapport entre la piscine et la nageuse (Birouste J., 1993). La relation que le sportif entretient avec son environnement est décrite ici avec une sensibilité et un langage entrant en harmonie avec la théorisation préalable qui se perçoit aussi dans l'anthropologie de Pierre Sansot.

La prise en compte de la dimension archéologique par Pierre Sansot ne signifie pas l'appel au passé comme explication du présent par la présence d'invariants, la permanence de l'archaïque est analysée davantage dans les transformations qu'il subit dans la modernité. Il s'agit alors, d'une conjugaison dans l'instant, de la mémoire en tant que celle -ci véhicule des signifiés différents de ceux que le présent convoque relativement à des signifiants semblables. Ici, la présence de l'ethnologie ou de l'histoire s'affirme et, si Gilbert Durand est très attentif aussi aux dissimultanéités dans la contemporanéité (1985), cette identité entre les deux auteurs n'est qu'apparente car Gilbert Durand entend par là appeler la Tradition contre la Raison pour valoriser ainsi l'imaginaire tandis que le questionnement du passé pour Pierre Sansot est une méthode de retour pour comprendre les transformations de la culture contemporaine. Un deuxième point semble encore différencier ces deux types de travaux: le poids à donner à la notion de structure au sein du schème herméneutique, car si le pôle structural n'est pas neutralisé dans les recherches de Pierre Sansot, puisque s'y affirme la notion de "forme", cela ne l'entraîne pas, comme Gilbert Durand à établir une classification. Comment comprendre cette divergence?

Le projet de l'archéologie anthropologique de Gilbert Durand est de construire, par la convergence, une classification des symboles constellant autour d'un même thème archétypal. Ce principe de convergence relève d'une homologie plutôt que d'une analogie, au sens où la convergence "joue davantage sur la matérialité d'éléments semblables plutôt que sur une simple syntaxe. L'homologie est l'équivalence morphologique, ou mieux structurale, plutôt qu'équivalence fonctionnelle (...). Ce sont ces ensembles, ces constellations où viennent converger les images autour de noyaux organisateurs que l'archéologie anthropologique doit s'ingénier à déceler à travers toutes les manifestations humaines de l'imagination" (1992: 41). L'aboutissement d'un tel désir organisationnel des symboles rompt cependant avec deux essais de classifications réalisés par Dumézil et par Piganiol qui insistaient le premier sur "le caractère fonctionnel et social des motivations du rituel, des mythes", le second "sur la différence des mentalités et des symbolismes qui découlent du statut historique et politique d'occupant ou d'occupé" (ibidem : 32), au sens où pour Gilbert Durand il s'agit là d'un positivisme objectif motivant les symboles par des données externes à la conscience imaginaire. La classification structurale des symboles de Gilbert Durand se 
fonde sur le rejet à la fois de l'idée d'une "totalitaire" infrastructure (ibidem : 496) et du projet "des psychophénoménologistes et les refoulements, ou les intimations sociofuges chères aux sociologues et aux psychanalystes" (ibidem: 37). Il souhaite accéder au contraire à une définition de la structure envisagée comme "une forme transformable, jouant le protocole motivateur pour un groupement d'images et susceptible elle-même de groupement en une structure plus générale que nous nommons Régime" (ibidem: 66).

Bien sûr, l'idée de "forme" est constante dans l'oeuvre de Pierre Sansot (et dans celle de Michel Maffesoli), et se trouve au principe du schème herméneutique (Berthelot J.-M., 1990 : 74), et il proclame sa volonté de ne pas souscrire au projet de découvrir les "structures anthropologiques". Alors, il semble bien que l'idée de "forme" prenne des sens variés selon les auteurs, les conduisant à des recherches empiriques contrastées. Autant Gilbert Durand s'accorde avec Claude Lévi-Strauss pour reconnaître le caractère insuffisant de la notion de forme qu'il considère comme une "abstraction sémiologique et figée" et préfère lui substituer la notion de structure par son caractère de "dynamisme transformateur" (ibidem: 66), autant les travaux de Pierre Sansot n'appellent pas le repérage de classifications ou de structures mais valorisent la notion de forme comprise cependant dans une dimension dynamique et ouverte à des pratiques variées ou de manière plus générale, à la culture de la vie quotidienne. Ainsi, si ces deux épistémologies s'accordent pour penser la notion de forme comme réunion de l'intelligible et du sensible, il semblerait qu'en lui substituant la notion de structure, Gilbert Durand la neutralise au nom de sa clôture, tandis que la même notion s'opérationnalise chez Pierre Sansot, dans une immédiateté saisissable des contenus empiriques, dans une configuration concrète dégagée d'une volonté de classification. Les formes révélées par Pierre Sansot sont buissonnières parce qu'elles concernent une pluralité d'objets apparemment séparés que la notion de forme réunit et organise, la dynamique structurale de Gilbert Durand converge vers une classification qui neutralise les contextes extérieurs obligatoirement changeants et fixe ainsi, probablement, la mouvance organisatrice, sans pour autant verser dans le schème d'intelligibilité structuraliste de Lévi-Strauss, puisque Gilbert Durand ne plaide jamais pour des modèles quantitatifs mais accorde plutôt "une primauté qualitative" aux "structures sémantiques" (ibidem: 66). Forme pour Pierre Sansot, structures pour Gilbert Durand, ces deux variantes se rapportent bien cependant au sens et non à des schèmes de nature causale ou fonctionnelle.

La sortie du cercle herméneutique

Pierre Sansot, à plusieurs occasions dans son oeuvre, prend de réelles distances à l'égard de la sociologie critique du sport qui s'idéologiserait en voulant démasquer les idées (1986 a : 82), car l'observation de ce phénomène excède les idéologies si on veut dévoiler des signes non lus. Par exemple, une pratique comme le Tour de France d'avant-guerre, pouvait certes raviver les nationalismes, sans s'y réduire cependant, au sens où ce même Tour, par les commentaires qu'il provoquait, révélait une "France fantastique, avec ses montagnes sacrées, comme l'Aubisque, le Tourmalet, des provinces comme les Flandres, le Limousin, des villages perdus dans le secret de leur pays. On voit donc que la fonction idéologique n'abolit pas nécessairement les rêveries collectives" (1991 b : 73). Pierre Sansot en voulant capter les rumeurs de la socialité (1986 a: 66) entend poser le problème d'une distinction au sein de l'imaginaire, "Faudrait-il donc démystifier au lieu de remythifier et distinguer deux sortes d'imaginaire inconciliables par leur approche et dans leur but? Un imaginaire qui, tout 
en ne relevant pas de la raison, invente des possibles, nous ouvre le chemin de la connaissance et de l'action - et un imaginaire qui se fiant à l'apparence et en la voulant belle parce qu'elle apparait, retarderait la prise de conscience de l'homme ?" (1973 : 418). Cette même distance est tenue par rapport à la sociologie de Pierre Bourdieu relativement à la culture, plus centrée sur la recherche des déterminants socioculturels et des luttes dans le champ qu'à un dévoilement rendu possible par une sociologie figurative plus proche du peuple comme l'a faite Hoggart. Les recherches de Jean-Marie Brohm comme celles de Pierre Bourdieu ont en commun, avec des variables (Le Pogam Y., 1983), de se construire sur une dénonciation de la logique de domination et Pierre Sansot, contre ces thèses entend bien donner des preuves à son approche, par la narration ou les descriptions. Il semble bien qu'il y ait là l'affirmation de deux types d'épistémologie correspondant à la défense du principe de démystification d'une part et à la nécessité de la présence de celui de "récollection" de sens d'autre part, comme l'a formulé Paul Ricoeur. Mais encore une fois, les oppositions entre ces deux manières de concevoir une "intelligence du social" sont-elles en tout point irréductibles? Jusqu'où va cette neutralisation du phénoménologique dans le pôle dialectique ou structural ? N'existe-t-il pas des convergences, notamment par le poids commun des affects, constitutifs de ces deux orientations que tout semble séparer? Comment alors interpréter la notion d'imaginaire social présente dans l'anthropologie poétique de Pierre Sansot et dans les théorisations des idéologies politiques, notamment par le poids que concèdent ces approches à la relation entre le sens et l'action, à la reconnaissance des symboles, à la prise en compte de la subjectivité dans les processus d'objectivation du social?

La sortie du cercle herméneutique

Précédemment, il a été dit que Pierre Sansot marquait une différence d'appréciation dans le schème herméneutique en se distanciant d'une possible organisation structurale des symboles. Dans sa construction de la réalité sociale, il relativise aussi la dimension de luttes de classes ou de rapports de force entre les groupes sociaux présents dans le champ, c'est-à-dire la dimension structurale des relations des acteurs en interaction, ramenée à des luttes symboliques et à des enjeux de pouvoir. La violence symbolique et la logique de domination ne sont pas inconnues de Pierre Sansot, mais sa poétique phénoménologique et la capacité structurante de l'imaginaire qu'il décrivait pour engendrer des symboles ou pour faire appel au sacré et aux rituels ne s'appliquent pas à ces directions. Bien qu'ancrée constamment au réel, sa pensée se différencie donc de celle de Pierre Bourdieu qui comme Gilbert Durand neutralise le structuralisme mécaniste de Claude Lévi-Strauss. Dans la logique de Pierre Bourdieu, les signes sont interprétés par leur pouvoir symbolique dépendant des échanges entre les acteurs dans le champ qui établissent des rapports de domination, et l'ordre symbolique est formé par l'ensemble des représentations qu'ils accordent aux pratiques, elles-mêmes liées aux dispositions des acteurs déterminées par des conditions objectives d'existence inégalitaires, ce qui génère des luttes de classe qui sont aussi des luttes pour un classement social, luttes produites par une liaison entre les structures objectives et cognitives (Bourdieu P., 1972).

La critique à la phénoménologie de Pierre Sansot lui sera adressée de deux manières : soit directement par les contre-critiques de J.-M. Brohm, soit indirectement par les réponses faites par Pierre Bourdieu quand il positionne ses théories au sein d'autres approches parmi lesquelles s'inscrivent la phénoménologie et l'ethnométhodologie. 
Ainsi, Pierre Sansot loue la finesse et la force des recherches de J.-M. Brohm sur l'institution sportive réalisée par la notion d'Appareil Idéologique d'État et fait part de son enchantement au regard de la cohérence du propos, mais il entend bien revendiquer comme recherche, le "résidu" non analysé par cette approche, ceci au moyen d'une pensée phénoménologique, approche que J.-M. Brohm va critiquer dans son fondement et dans sa méthode. Pour lui, l'analyse du sport ne peut en effet mettre entre parenthèses les déterminants socioculturels, ni les descriptions parvenir à mieux comprendre la réalité qu'une approche conceptuelle, car elles tendent à en donner des fragments inessentiels du phénomène, survolé alors de façon impressionniste, phénomène qui se donne à comprendre dans sa genèse, sa structure et son fonctionnement et à lire dans la totalité du système social. Enfin si un regard empirique se manifeste obligatoirement, il doit être construit et ne pas être constitué de "simples descriptions" et comme tel, il ne peut rendre compte de la totalité dynamique de la réalité (1993 : 177 sq.).

Ce type de critiques s'organise contre ce qui est pensé comme étant une réduction opérée par la phénoménologie de Pierre Sansot qui relativise dans le schème herméneutique l'ancrage objectiviste, tout comme les analyses de la culture de Pierre Bourdieu se fondent sur ce que les approches de Pierre Sansot neutralisent, c'est-à-dire la présence dans le pôle phénoménologique du schème structural. Dès Esquisse d'une théorie de la pratique (1972), Pierre Bourdieu entendait déjà fonder une connaissance du social sur la nécessité d'une analyse praxéologique comme dépassement et intégration de l'objectivisme (les conditions matérielles d'existence) et de la phénoménologie (le constructivisme des formes cognitives). Il ne cessera d'amplifier sa relativisation de la phénoménologie ou de l'ethnométhodologie, au sens où il faut "sociologiser la doxa" (1992: 52), en échappant à une analyse du sujet, sans sacrifier l'agent "à la philosophie de la structure mais sans renoncer à ses effets" (ibidem : 97) et en ne donnant alors la priorité, ni à l'acteur ou à la structure, mais aux relations (champ), et en dégageant la signification politique de la sociologie par les logiques de domination révélées par les rapports de force des agents qui structurent le champ. Ainsi se comprend toute la différence entre l'adhésion de Pierre Bourdieu au "structuralisme" de Goffman et le rejet en cet auteur de ce même aspect par Pierre Sansot. Sans doute les différences entre Pierre Bourdieu et Pierre Sansot peuvent-elles être appréciées par un exemple d'analyse et d'interprétation d'un même phénomène de la vie quotidienne, celui d'un bal de campagne et des bals du 14 Juillet, deux faits inscrits dans la culture populaire.

87 Pierre Bourdieu décrit un petit bal de campagne dans le Béarn à la veille de Noël en évitant l'emploi du "je", et montre comment la compréhension du défi entre les citadins qui dansaient et les célibataires plus âgés qui ne dansaient pas et qui se rapprochaient du centre, l'invite à une analyse statistique du taux de célibat et des stratégies matrimoniales et à mettre en exergue la notion de violence symbolique rompant avec le marxisme qui a été aveugle à ces violences douces et avec la théorie de Foucault de la domination comme discipline ou dressage, pour conclure qu'elle est "plus redoutable que la violence politico-policière, parce qu'elle est méconnaissance fondée sur l'ajustement inconscient des structures subjectives aux structures objectives" (ibidem : 141-142). Le bal du 14 Juillet décrit par Pierre Sansot par opposition aux fêtes "officielles" est interprété comme une fête populaire appréciée par des "menus signes qui relèvent de la sphère sensorielle", le feu d'artifice qui "touche" les 
foules, la vue (les drapeaux aux fenêtres qui donnent un caractère festif), le vacarme et les bruits qui composent un espace commun, (l'accordéon), la tactilité (contacts furtifs ou prolongés, l'enlacement des couples, les bagarres), les odeurs (des foules, de la valse, de la bière, les fumets), le goût (les banquets), la présence des corps (défis de force ou d'adresse, mât de cocagne), bref une "fête populaire" qui "atteint rarement chez nous l'état de transe" (1991 a : $175 \mathrm{sq}$.). C'est encore du poids démonstratif de la description dont il est question ici et de la valeur intrinsèque de celle-ci, même si Pierre Sansot reconstruit le sensible en l'organisant. Ce que pense Pierre Bourdieu à ce sujet est éloquent: "Je conviens qu'il y a une expérience première du social qui, comme l'ont montré Husserl et Schütz, repose sur un rapport de croyance immédiate qui nous porte à accepter le monde comme allant de soi. Cette analyse est excellente en tant que description, mais il faut aller au-delà de la description et poser la question des conditions de possibilité de cette expérience doxique. On voit alors que la coïncidence entre les structures objectives et les structures incorporées, qui crée l'illusion de la compréhension immédiate, est un cas particulier dans l'univers des relations possibles au monde, celui de l'expérience indigène. La grande vertu de l'expérience ethnologique est ici qu'elle fait immédiatement découvrir que ces conditions ne sont pas universellement remplies, comme le fait croire la phénoménologie lorsqu'elle universalise (sans le savoir) une réflexion fondée sur le cas particulier de la relation originaire du phénoménologue à sa propre société" (1992: 52-53). On le voit, le "je" n'est pas de mise dans cette approche et la théorie de la connaissance du social requiert l'analyse des conditions objectives qui rendent le pensé pensable. Le relationnisme méthodologique de Pierre Bourdieu neutralise la priorité à accorder au sujet pour l'intégrer dans la matérialité, comme il le suggère à propos de la relation entre l'habitus et le champ, "(...) c'est une véritable complicité ontologique qui, comme Heidegger et Merleau-Ponty le suggéraient, unit l'agent (qui n'est ni un sujet ni une conscience, ni un simple exécutant d'un rôle ou l'actualisation d'une structure ou d'une fonction) et le monde social (qui n'est jamais une simple chose même s'il doit être construit comme tel dans la phase objectiviste de la recherche" (ibidem : 103).

Enfin, il est un autre point de désaccord entre ces deux sociologies de la culture populaire, l'interprétation du langage. On sait toute l'importance que lui prête Pierre Sansot dans la pensée herméneutique. Pierre Bourdieu se distancie encore d'une approche qui néglige le langage comme support de pouvoir, "Le point de vue saussurien est celui d'un "spectateur impartial" qui cherche la compréhension pour la compréhension et qui est ainsi conduit à prêter cette "intention herméneutique" aux agents sociaux, et à en faire le principe de leurs pratiques (...). Ceux qui traitent le langage comme un objet d'analyse au lieu de l'utiliser pour penser et pour parler, sont portés à le constituer comme un logos, par opposition à une praxis, comme une lettre morte sans fin pratique ou sans autre dessin que celui d'être interprétée, à la manière de l'oeuvre d'art (...). Le langage, tant chez Saussure que dans la tradition herméneutique, est traité comme lettre morte (écrite ou étrangère, comme dit Bakhtine), comme système autosuffisant complètement coupé de ses fonctions pratiques et politiques (comme dans la sémantique pure de Fodor et Katz)" (1992 : 116). Ici, comme pour la culture, le langage n'a de réalité que considéré dans des rapports de force exprimant des rapports de domination, dimension niée par les ethnométhodologues qui les accepteraient comme réalités immédiates.

Convergences herméneutiques? 
Il pourrait paraître audacieux d'espérer trouver quelques convergences entre ces deux herméneutiques, celle de la mystification et celle de la restauration de sens, tant les schèmes d'intelligibilité qui les construisent paraissent éloignés. Ici en effet, la logique structurale, présente déjà dans les travaux de Gilbert Durand les différenciant de ceux de Pierre Sansot, est poussée à son extrême par son intégration à des conditions sociologiques, historiques et concrètes, par une logique de la domination propre aussi à la sociologie marxiste. Ainsi s'opère entre les théories, "une matrice d'échanges généralisés" de schèmes explicatifs (Berthelot J.-M., 1990, 103) qui ont été opérationnalisés pour maximiser les différences. Mais n'est-il pas possible aussi de voir des schèmes explicatifs semblables dans des théories différentes, ce qui contribuerait à minimiser les écarts entre elles, sans que leur singularité soit affectée?

Ainsi Pierre Bourdieu croit à la phénoménologie comme il a été vu, et sa définition d'une classe sociale par exemple se fonde en partie sur la représentation qu'en ont les agents, au-delà des caractéristiques objectives et en ce sens, l'univers des significations immédiates n'est pas déconsidéré. D'autre part, vis-à-vis du langage, on trouve dans l'anthropologie de Pierre Bourdieu, comme dans celle de Pierre Sansot, le même goût pour la déconstruction des mots: chacun n'engage une connaissance du social qu'en ayant au préalable mis en question les "productions matérialisées" que la sociologie emploie comme instruments de construction, comme les CSP de L'INSEE qui apparaissent pour Pierre Bourdieu comme une "conceptualisation bureaucratique" (1992 : 212), et Pierre Sansot aime à se livrer à une déconstruction de la notion de classe de Pierre Bourdieu afin d'appréhender "les gens de peu" (1991), et face aux catégories toutes faites, vise dans une page exemplaire, à "arracher à l'INSEE" le terme "ménage" afin d'évoquer une "socialité confuse, rebelle à toute conceptualisation" (ibidem: 69-70). Pierre Sansot discutera encore la notion de marginalité en hommage à Yves Barel qui l'avait interprétée comme la crise de sens née d'un vide social, et il pose la question - tout en admettant qu'il y eût vide social du sens - de savoir malgré tout si nous ne continuons pas "de notre condition d'être sentant, de notre corps agissant - à instituer le monde, du plus élémentaire au plus noble, du plus élémentaire c'est-à-dire avec un horizon, une division selon le proche et le lointain, le bas et le haut ?" (1993 d : 162). Enfin, il faudrait souligner le goût commun qu'ont ces deux auteurs pour la philosophie, notamment phénoménologique, dont on imaginerait mal qu'il n'ait pas d'incidence pour construire leur théorie du social, car elle s'attache au "très près", sans pour autant que leurs recherches puissent être qualifiées de philosophiques. Pierre Bourdieu en effet, ne voit pas "une différence ontologique entre le concept de Kant ou de Platon et une analyse factorielle" (1992: 133), et l'oeuvre de Pierre Sansot multiplie aussi les enquêtes, les hypothèses, différenciant ainsi ses recherches d'une pure philosophie malgré son attachement aux phénoménologues. Enfin, tous deux se nourrissent bien des écrivains et des poètes parce qu'ils reconnaissent le rôle de l'intuition dans la créativité sociologique et dans la formalisation du réel, "C'est par là, écrit Pierre Bourdieu, que le travail du sociologue s'apparente au travail de l'écrivain (je pense à Proust): comme lui, nous avons à faire accéder à l'explication des expériences génériques ou spécifiques, qui, à l'ordinaire, passent inaperçues ou restent informulées" (ibidem: 178). Littérature et sociologie ne s'opposent pas dans ces deux types d'épistémologie, l'oeuvre littéraire peut être source d'orientation de recherche, et tous deux rompent avec une perception naïve de la littérature en la faisant passer par le rang de la science, sans ignorer les divergences des genres, même si l'écriture de 
Pierre Sansot poétise la réalité et contribue à une approche figurative des manières de faire non pervertie par le langage scientifique.

91 Enfin, il faudrait poursuivre cette recherche de ressemblances dans des pensées antagonistes par d'éventuels liens entre l'imaginaire collectif développé par Pierre Sansot, et la sociologie des imaginaires sociaux construite sur la prise en compte des idéologies politiques dont il se sépare volontairement. Ici encore, quelle que soit l'opposition des champs d'études, et notamment la prise en compte du politique, il s'agit dans les deux directions, de rompre avec le positivisme scientiste et avec une conception de l'imaginaire pensé comme une puissance illusoire ou une ornementation des rapports économiques ou politiques. L'imaginaire contribue dans les deux cas à une dynamique instituante.

Les travaux de Pierre Ansart s'attachent à faire le lien entre les imaginaires sociaux et les idéologies politiques. Les imaginaires sociaux supposent l'adhésion à deux hypothèses: la cohérence d'un groupe ou d'une classe se situe dans des images, "symbolisation du réel et de l'irréel, les constructions du passé et du futur, les significations artistiques" et cela s'inscrit dans des actions sociales (1984). Les idéologies, quant à elles ne sont pas des représentations tronquées mais plus exactement des "ensembles symboliques en évolution à travers lesquels le groupe se redéfinit, s'identifie, se met en scène et projette ses rêves. L'idéologie n'est pas seulement un sujet de représentations déformantes, mais le lieu où s'investissent les espoirs et les craintes, les fantasmes et les angoisses" (1979: 7). Le rôle des idéologies politiques est de transformer l'imaginaire. L'idéologue induit un ensemble de conséquences symboliques et pratiques. Ici donc, concernant les idéologies politiques, l'imaginaire se construit aussi sur une vue que ne renierait probablement pas Pierre Sansot, c'est-à-dire la révélation d'une dimension occultée par une tradition sociologique et qui éclaire, par une approche clinique, l'essence et les fonctions de l'idéologie montrant "l'inachèvement, la fragilité, le caractère approximatif de toute formation sociale" (Ansart P., 1977 : 105). Bien sûr, malgré cette convergence, Pierre Sansot ne privilégie pas les processus conflictuels mais il contextualise bien les émergences de l'imaginaire, car si l'imaginaire est le produit de représentations collectives auxquelles s'attachent par exemple les légendes qu'il décrit, ces représentations se cristallisent bien aussi à partir de contextes dont les propriétés sont changeantes, une société ne produit pas dans le temps des héros identiques. En ce sens l'imaginaire social auquel il se réfère est génératif, comme l'est l'imaginaire social travaillé par les idéologies politiques ou les espoirs collectifs (Baczko B., 1984), qui sont symboliques parce qu'ils s'ancrent eux aussi dans la puissance des affects. Dans ces deux cas, le social n'apparaît pas comme un "donné", il n'est pas substantialisé. L'imaginaire est aussi poiétique et dans une logique toute wébérienne, il crée du sens lié à une action. Ce qui semble alors différencier l'imaginaire de Pierre Sansot des imaginaires sociaux ou idées-images qu'une société crée et renouvelle, ce sont les champs d'analyse et l'appel aux théories qui les construisent. Au-delà en effet de l'appel à Weber pour donner sens à une herméneutique, Pierre Ansart convoque Marx, à condition de le lire dans la démonstration qu'il fait de "l'efficacité historique des imaginaires" et de s'éloigner d'une vue positive de l'histoire, comme le montre l'analyse du 18 Brumaire de Louis Bonaparte, conditions qui font que l'imaginaire social peut devenir "une force matérielle et déterminer les comportements des acteurs" (1984). Il devient instituant comme le suggérait Pierre Sansot, simplement le champ de lecture s'est déplacé aux pratiques sociales et politiques avec un regard identique 
réfutant toute interprétation mécaniste des idéologies (Ansart P., 1968). En cela donc des sensibilités communes apparaissent, malgré des lectures de l'imaginaire réalisées dans des registres que tout sépare a priori. Le projet en effet de Pierre Ansart recoupe celui de Pierre Bourdieu, au sens où il s'appuie sur un schème structural car l'interprétation des idéologies impose une problématique des conflits, de positions des groupes, d'insertion sociale, visant à multiplier les liens entre les discours et les agents sociaux, à restituer les structures, les divisions, les enjeux et les stratégies qui sont le sous-jacent du signifiant et qui permettent de comprendre et d'expliquer (1974: 6-7). Les idéologies, les mythes et les utopies font les imaginaires sociaux qui reposent sur un symbolisme contribuant à l'inachèvement du social, en dépassant une vue fonctionnaliste, puisque l'efficace du symbolique tient à l'intégration de la dimension d'opposition.

Cette volonté de trouver quelques convergences ne saurait masquer la singularité des auteurs présents dans le champ de l'herméneutique superficiellement abordés ici. Pierre Sansot en ce sens témoigne d'un goût affirmé pour l'analyse de "fragments" de la vie sociale, contre les sociologies globalisantes. C'est là le choix d'une vue quelque peu simmelienne sur laquelle réfléchit Marc Guillaume et qui semble-t-il pourrait s'appliquer à Pierre Sansot, vue attachée à "comprendre l'univers intime où se nouent les liens affectifs et passionnels selon une symbolique spécifique", position dangereuse car l'observation sur laquelle il s'appuie "porte le sujet à son point d'ébullition comme le disait Georges Bataille", auteurs qui sont aussi des "acteurs" au sens où "Ils n'agissent pas seulement en produisant un plus de savoir ou d'utilité, ils produisent une théorie, c'est-à-dire une certaine façon de jouer le destin social. Ce jeu échappe totalement à la dialectique du vrai et du faux, il affranchit la réalité humaine des principes de rationalité. L'important est qu'il nous séduise (...). De même, les poètes du social nous proposent leurs visions du monde tel qu'il n'est pas mais tel qu'il pourrait être, si, séduits, nous observions des règles qui, pour devenir effectives, n'attendent que d'être dites" (Guillaume M., 1989 : 215 sq.).

Pierre Sansot prête à ses rêveries une puissance et un pouvoir dans la quête du sens du monde social. Tel un funambule, il se risque dans des voies tendues par un fil théorique et méthodologique dont il sait la fragilité malgré sa défense argumentée et la logique de la preuve qu'il apporte, fragilité car la science se positionne dans un champ dont les enjeux sont aussi sociaux. Cet équilibre qui paraît délicat dans ses analyses est en même temps fascinant, car il l'oblige à des figures originales se démarquant des voies balisées et banalisées des écoles de pensée les plus reconnues. Pour cela, il est capable de ruser : il se sert de son propre émerveillement qu'il nous transmet pour révéler des situations ou des personnages de la vie quotidienne que nous ne savons plus observer et qui se détachent de nous, nous renvoyant par là à notre propre ignorance. En nous invitant dans ses voyages et en faisant appel aux imaginaires, il nous entraîne sur des voies inconnues et des découvertes révélées par le sensible. En ce sens, il recoupe bien le projet des sciences sociales: mettre ses instruments, ici une herméneutique phénoménologique au service d'une liberté intellectuelle.

Evidemment une telle quête du plaisir peut paraître cruelle et décalée dans un monde où d'autres préfèrent décrire la misère et la domination. Mais Pierre Sansot n'ignore pas celles-ci, ni les déterminants économiques, les tenants et les aboutissants de la sociologie politique, les oppositions ou les enjeux dans le monde social. Il les tient seulement à distance, imaginant qu'ils sont bien présents dans les formes qu'il décrit, 
mais en les surplombant, "Si nous n'oublions pas les génocides, les guerres, les crises, la fureur criminelle de certains individus, la misère abominable des peuples entiers, nous sommes en droit de composer la plus belle légende du siècle et de nous livrer au jeu poétique des associations " (1993 b : 189). D'autre part si la science dans son caractère objectif lui apparaît tout à fait recevable, elle est aussi neutralisée au sens où le monde peut être révélé par le mode du sensible, "En fin de compte et pour notre bonheur, nous pouvons vivre selon deux registres. Le même individu peut à la fois penser selon les procédures de la science et exister sur un mode naif. En tant que physicien, il sait très bien quel la terre tourne, mais, en tant qu'être vivant, sensible, pour lui, comme Husserl le rappelait, la terre ne tourne pas rond. Chaque matin, il voit le soleil se lever (...). Cette immédiateté ne nous empêchera pas d'emprunter le chemin ardu des médiations, des concepts, du recours aux archives... et d'y trouver un plaisir qui s'ajoutera à d'autres bonheurs ou qui les surmontera, si l'on veut bien admettre que la passion de comprendre constitue la plus pure et la plus noble des joies" (1992 a: 195). Il est alors salutaire de voir le champ des sciences de l'homme se complexifier par des regards contradictoires portés sur la réalité sociale, signe de la fécondité de la recherche, sans qu'il faille établir entre eux une quelconque hiérarchie construite sur la prétendue "vérité" que certaines détiendraient par rapport à d'autres. Penser hors normes, c'est penser qu'une connaissance du social puisse être établie, non pas par d'autres schèmes d'intelligibilité que ceux dont héritent les sciences sociales ou de ceux qu'elles créent, mais par le bouleversement de formes de pensée apporté par la combinatoire de ces schèmes, dans un champ déjà constitué, non préparé à les recevoir. La phénoménologie poétique de Pierre Sansot constitue ce moment de tension dans un champ où dominent parfois des "concepts cloutés" (Laplantine F. 1994) et un langage spécialisé pour les opérationnaliser. Parvenir à de "simples descriptions" qu'accompagne une écriture "lyrique" (1994 b : 66). tend à laisser croire qu'elles ne révèlent plus rien d'essentiel, qu'elles ne seraient qu'une étape d'une recherche qui irait plus loin, alors qu'il s'agit là de notre premier regard avec le réel et cela participe alors d'une tâche bien plus risquée que la démonstration standardisée qui ne surprend plus tant le moule méthodologique est universalisé. La démarche de Pierre Sansot est résolument poétique et ne peut s'accorder avec la recherche de lois, de causes ou de structures et sans doute s'accorderait-il avec le poète Milosz qui définissait la poésie comme "une poursuite passionnée du Réel", et qui la réalise avec une écriture faite avec "l'âme des mots" ?

\section{BIBLIOGRAPHIE}

Ansart P. (1968).- Marx et la théorie de l'imaginaire social, Cahiers Internationaux de Sociologie, vol XLV, p. 96-116.

Ansart P. (1972).- L'occultation idéologique, Cahiers Internationaux de Sociologie, vol LIII, p. 213-228.

Ansart P. (1974).- Les idéologies politiques, Paris, PUF.

Ansart P. (1977).- Idéologies, conflits et pouvoir, Paris, PUF. 
Ansart P. (1979).- Eléments d'épistémologie pour une sociologie des affects politiques, Document ronéotypé, Paris VII.

Ansart P. (1983).- La gestion des passions politiques, Lausanne, L'âge d'homme.

Ansart P. (1984).- L'imaginaire social, Encyclopaedia Universalis, Supplément Les Enjeux, p. 710-713.

Ansart P. (1990).- Les sociologies contemporaines, Paris, Ed. du Seuil, Coll. Points, 211.

Aubert F. (1992) (Textes réunis par).- Variations sociologiques. En hommage à Pierre Ansart,

L'Harmattan.

Augé M. (1982).- Football. De l'histoire sociale à l'anthropologie religieuse, Le Débat, 19, 59-67.

Bachelard G. (1942).- L'Eau et les rêves. Essai sur l'imagination de la matière, Paris, Librairie José de Corti.

Bachelard G. (1943).- L'Air et les songes. Essai sur l'imagination du mouvement, Paris, Librairie José de Corti.

Bachelard G. (1975).- La formation de l'esprit scientifique, Paris, Vrin.

Baczko B. (1984).- Les imaginaires sociaux. Mémoires et espoirs collectifs, Paris, Payot.

Balandier G.(1974).- Anthropo-logiques, PUF.

Balandier G. (1993).- Sensuelle et rêveuse sociologie, Le Monde, 26 mars.

Bataille G. (1967).- La Part maudite. Précédé de La Notion de dépense, Paris, Ed. de Minuit.

Berthelot J-M. (1990).- La connaissance du social. Le pluralisme explicatif en sociologie, Paris, PUF.

Birouste J. (1993).- Pour une étude de l'aesthésique sportive, Sciences Humaines Cliniques, Tome 2, Quel Corps ? 45-46, 204-211.

Bourdieu P. (1972).- Esquisse d'une théorie de la pratique, Genève, Droz.

Bourdieu P. (1989).- La Distinction, Paris, Ed. de Minuit.

Bourdieu P., Wacquant L. J.D. (1992).- Réponses. Pour une anthropologie réflexive, Paris, Le Seuil.

Bromberger C. (1987).- La passion du football à Marseille et à Turin, Terrains, 8, 8-41.

Brohm J-M. (1982).- Corps et pouvoir, Actions et recherches sociales, 1.

Brohm J-M. (1993).- Les meutes sportives. Critique de la domination, Paris, Ed. L'Harmattan.

Brown R. (1989).- Clefs pour une poétique de la sociologie, Arles, Ed. Actes Sud.

Canguilhem G. (1957).- Sur une épistémologie concordataire, in Hommage à Bachelard, Etudes de philosophie et d'histoire des sciences, Paris, PUF, p. 3-12, cité par Bourdieu P., Chamboredon J.-C., Passeron J.-C., (1973), Le métier de sociologue, Paris, Mouton/Bordas, p. 114.

Corbin H. (1976).- L'imagination créatrice dans le soufisme d'Ibn' Arabî, Paris, Flammarion (1958).

Dufrenne M. (1973).- Préface à Poétique de la ville, Paris, Ed. Klincksieck, 3-5.

Dumazedier J. (1974). -Sociologie empirique du loisir, Paris, Ed. du Seuil.

Dumazedier J. (1988).- Révolution culturelle du temps libre, 1968-1988, Paris, Ed. Klincksieck.

Durand G. (1979).- Sciences humaines et tradition, Berg International Editeurs (1975).

Durand G. (1984).- L'Imagination symbolique, Paris, PUF (1964). 
Durand G. (1984).- Entretiens avec Le Monde (réalisé par Christian Descamps le 15 juin 1980, "Gilbert Durand, géographe de l'imaginaire"), in Civilisations, T4, Paris, Ed. La Découverte/Le Monde, p. 120-130.

Durand G. (1985).- Une réponse à la sociologie française, in Une anthropologie des turbulences, Berg International Editeur, p. 26-43.

Durand G. (1992).- Les structures anthropologiques de l'imaginaire. Introduction à une archétypologie générale, Paris, Ed. Dunod (Bordas, 1969, 1ère édition).

Duvignaud J. (1985).- Après le fonctionnalisme et le stucturalisme, quoi ? in Une anthropologie des turbulences, Berg International Editeur, p. 151-162.

Duvignaud J. (1991).- Le sport dans la société industrielle, in Anthropologie du sport. Perspectives critiques, Paris, Ed. AFIRSE/Quel Corps, p. 38-42.

Ehrenberg A. (1988).- L'âge de l'héroïsme. Sport, entreprise et esprit de conquête dans la France contemporaine, Cahiers Internationaux de Sociologie, LXXXV, 197-224.

Guillaume M. (1989).- La contagion des passions. Essai sur l'exotisme intérieur, Paris, Plon.

Habermas J. (1987).- A propos de la problématique de compréhension du sens dans les sciences praxéologiques empirico-analytiques, in Logique des sciences sociales et autres essais, Paris, PUF, p. $118-215$.

Jauss H.-R. (1987).- Pour une esthétique de la réception, Paris, Gallimard (1978).

Jeu B. (1977).- Le sport, l'émotion, l'espace. Essai sur la classification des sports et ses rapports avec la pensée mythique, Paris, Ed. Vigot.

Jeu B. (1992 a).- Violences, in L'histoire en mouvement. Le sport dans la société française (XIXe-XXe siècle) (sous la direction de Hubscher R.), Paris, Ed. Armand Colin, p. 379-399.

Jeu B. (1992 b).- L'exploitation du mimodrame tragique, in L'histoire en mouvement. Le sport dans la société française (XIX ${ }^{e}-X^{e}$ siècle) (sous la direction de Hubscher R.), Paris, Ed. Armand Colin, p. 401-417.

Jeu B. (1992 c).- Champions, championnes, in L'histoire en mouvement. Le sport dans la société française (XIX $-X X^{e}$ siècle) (sous la direction de Hubscher R.), Paris, Ed. Armand Colin, p. 419-438.

Laplantine F. (1994).- Epistémologie de la séparation et épistémologie de la traduction.Prétentaine, L'intellectuel dans la cité, 2-3, décembre, p. 156.

L'Express (1994).- Révolution dans le monde du rugby, 2259, 20 octobre.

Le Pogam Y. (1983).- Sport(s) et lutte de classes, Sports et sociétés contemporaines, VII Symposium de l'I.C.S.S, Paris, Société Française de Sociologie du Sport, p. 513- 525.

Maffesoli M. (1976).- Logique de la domination, Paris, PUF.

Maffesoli M. (1982).- L'ombre de Dionysos, Paris, Méridiens/Anthropos.

Maffesoli M. (1984).- Vie publique. Vie privée, Encyclopaedia Universalis, supplément Les Enjeux, p. 692-695.

Maffesoli M. (1985 a).- Pour une sociologie relativiste, Cahiers Internationaux de Sociologie, vol LXXVII, p. 5-13.

Maffesoli M. (1985 b).- La société est plusieurs, in Une anthropologie des turbulences, Berg International Editeur, p. 175-180. 
Maffesoli M. (1985 c).- La connaissance ordinaire. Précis de sociologie compréhensive, Paris, Librairie des Méridiens.

Maffesoli M. (1988).- Le temps des tribus. L'individualisme dans les sociétés de masse, Paris, Méridiens/ Klincksieck.

Maffesoli M. (1990).- Au creux des apparences. Pour une éthique de l'esthétique, Paris, Plon.

Maffesoli M. (1991).- Implosion de la société programmée, Commentaire, 55, p. 529-532.

Maffesoli M. (1992).- La solidarité post-moderne, Pour, 135, septembre, p. 59-64.

Maffesoli M. (1992).- La transfiguration du politique, Paris, Grasset.

Nahoum-Grappe V. (1993).- L'ingouvernable gratuité : les conduites de vertige, Communications, 56, (Le gouvernement du corps), p. 155-173.

Pociello C. (1983).- Le Rugby ou la guerre des styles.- Paris, A.-M. Métailié.

Raspaud M. (1983).- Football, rites, fondation : l'instant du match, Thèse de $3^{\mathrm{e}}$ cycle de Sociologie, Grenoble.

Ricoeur P. (1965).- De l'interprétation. Essai sur Freud, Paris, Ed. du Seuil.

Sansot P. (1973).- Poétique de la ville, Paris, Ed. Klincksieck.

Sansot P., Strohl H., Torgue H., Verdillon C., (1978).- L'espace et son double. De la résidence secondaire aux autres formes secondaires de la vie sociale, Paris, Ed. du Champ urbain.

Sansot P. (1980).- Variations paysagères, Paris, Méridiens/Klincksieck.

Sansot P. (1985).- La France sensible, Seyssel, Champ-Vallon.

Sansot P. (1986 a).- Les Formes sensibles de la vie sociale, Paris, PUF (contient un chapitre "Vers une sociologie des émotions sportives", p. 63-103, version élargie de "Une sociologie des émotions sportives", Cahiers Internationaux de Sociologie, vol. LXVII, 1984, p. 323-338).

Sansot P., Leroux H. (1986 b).- La pensée interprétative, in Guillaume M. (sous la direction de), L'état des sciences sociales, Paris, Ed. La Découverte, p. 39-42.

Sansot P. (1988).- Eloge de la parole sportive ; un exemple privilégié : le rugby, in Michon B., Faber C. (Textes réunis par), Sciences sociales et sports, Etats et perspectives, Actes des journées d'études de Strasbourg, Université des Sciences Humaines/Laboratoire APS et Sciences Sociales UFRSTAPS, p. 267-273.

Sansot P. (1989 a).- Cahiers d'enfrance, Champ-Vallon.

Sansot P. (1989 b).- Le respect des convenances, Conférence, Montpellier.

Sansot P. (1989 c).- Ode au sensible, Conférence, Montpellier.

Sansot P. (1990 a).- Le Rugby est une fête au pays de l'ovale, Paris, Plon.

Sansot P. (1990 b).- Quel salut attendre du sport ? , in Rivière C., Piette A. (sous la direction de), Nouvelles idoles et nouveaux cultes, Dérives de la sacralité, Paris, Ed. L'Harmattan, 59-75.

Sansot P. (1991 a) .- Les Gens de peu, Paris, PUF (contient un chapitre sur "Le football des trottoirs", p. 141-154, "Le roi des carreaux", p. 155-164, "La légende du Tour de France", p. 189-204, publié dans une première version sous le titre "Le Tour de France : une forme de liturgie", in Cahiers Internationaux de Sociologie, vol LXXXVI, p. 91-105). 
Sansot P. (1991 b).- Une forme d'imagination créatrice : l'imaginaire sportif, in Ardoino J., Brohm $\mathrm{J}-\mathrm{M}$ (sous la direction de), Anthropologie du sport, Perspectives critiques, Actes du Colloque international francophone Paris-Sorbonne, ANDSHA/Matrice/Quel Corps ?, p. 71-75.

Sansot P. (1992 a).- Un autre regard : à défaut de cet absolu qui se dérobe à l'homme ..., in Le corps surnaturé, Les sports entre science et conscience, Ed. Autrement, 4, p. 179-195.

Sansot P. (1992 b).- Papiers rêvés, papiers enfuis, Ed. Morgana.

Sansot P. (1993 a).- Jardins publics, Paris, Payot.

Sansot P. (1993 b).- L'imaginaire : la capacité à outrepasser le sensible, Sociétés, Approches méthodologiques, 42, Ed. Dunod, p. 411-417.

Sansot P. (1993 c).- La légende des sports, Communication au Colloque Sport, Culture, Tradition, Agde.

Sansot P. (1993 d).- Le marginal et l'existant, in Système et paradoxe, Autour de la pensée d'Yves Barel (Textes rassemblés par Michel Amiot, Isabelle Billiard et Lucien Brams), Ed. du Seuil, 1993, p. 159-168.

Sansot P. (1994 a).- Les Pilleurs d'ombres, Paris, Payot.

Sansot P. (1994 b).- Le goût de l'écriture : une dérive épistémologique ?, Communications, L'écriture et les sciences de l'homme, 58, 1994, p. 61-67.

Sfez L. (1978).- L'enfer et le paradis, Paris, PUF.

Simmel G. (1981).- Sociologie et épistémologie, Paris, PUF (1970).

\section{RÉSUMÉS}

L'oeuvre de Pierre Sansot occupe une place majeure et singulière dans le paysage de l'anthroposociologie contemporaine. Majeure par son épistémologie qui lui permet de scruter des objets très différents - dont le sport - en leur conservant une unité d'analyse, singulière par sa méthode qui l'autorise à faire émerger un sens inédit aux pratiques de la vie quotidienne. Il s'agira ici d'ordonner quelques éléments théoriques propres à l'auteur et de montrer leur fécondité quand ils s'attachent à décrire les êtres et leurs pratiques révélés alors dans une dimension insoupçonnée.

Fondée sur une approche phénoménologique, l'observation de la vie sociale portée par le sensible laisse alors apparaître tout le poids de l'imagination qui conquiert une légitimité et confère aux recherches un nouveau statut: celui d'un "réalisme poétique", c'est-à-dire la possible conciliation entre la science et la poésie. Deux directions souvent antagonistes qui entrent ici en harmonie grâce à une écriture resserrant cette alliance.

The works of Pierre Sansot take a great and singular position in the contemporary anthroposociology scene. Great, with his epistemology that scans very different objects- including sportkeeping the analysis unity, and singular, with a method that permits the apparition of a new signification of everyday life practices. We'll present here some theoretical elements proper to the author, and show their fertility when they describe human being and their practices both revealed with a new dimension.

Based on a phenomenological approach, the social life observation with a sensitive way lets appear all the importance of imagination, that gets some legitimacy and gives a new statute to researches: a poetic realism, i.e. the possible conciliation between science and poetry. These 
directions, often opposed, are here in harmony because of the writing that makes this alliance possible.

INDEX

Keywords : sport, imagination, poetry, sensitive

Mots-clés : poésie, sensible

\section{AUTEUR}

YVES LE POGAM

UFR STAPS Montpellier, Equipe "Corps et Culture" 